\title{
Assessment of wavelength-dependent parameters of photosynthetic electron transport with a new type of multi-color PAM chlorophyll fluorometer
}

\author{
Ulrich Schreiber • Christof Klughammer • \\ Jörg Kolbowski
}

Received: 8 March 2012 / Accepted: 8 June 2012/Published online: 23 June 2012

(c) The Author(s) 2012. This article is published with open access at Springerlink.com

suspensions of unicellular algae and cyanobacteria. Plots of ETR(II) versus PAR(II) for Chlorella are almost identical using either 440 or $625 \mathrm{~nm}$ light. Photoinhibition data are presented suggesting that a lower value of ETR(II) $\max$ with $440 \mathrm{~nm}$ possibly reflects photodamage via absorption by the Mn-cluster of the oxygen-evolving complex.

Keywords Chlorella $\cdot$ ETR $\cdot$ Functional absorption cross section of PS II · $O-I_{1}$ fluorescence rise · PAR ·

Photoinhibition · Synechocystis

$\begin{array}{ll}\text { Abbreviations } \\ \text { AL } & \text { Actinic light } \\ \text { COB } & \text { Chip on board } \\ \text { ETR } & \text { Electron transport rate } \\ \text { ETR(II) } & \text { Absolute rate of PS II turnover, electrons/ } \\ & \text { (PS II s) } \\ F_{\mathrm{m}} & \text { Dark-acclimated maximal fluorescence yield } \\ F_{\mathrm{m}}^{\prime} & \text { Maximal fluorescence yield during } \\ & \text { illumination } \\ F_{\mathrm{o}} & \text { Dark-acclimated minimal fluorescence yield } \\ \text { FR } & \text { Far-red light ( }>700 \text { nm) } \\ \text { FRR } & \text { Fast repetition rate } \\ F_{\mathrm{v}} & \text { Variable fluorescence yield } \\ I_{k} & \text { Light intensity at onset of saturation ( } \mu \text { mol } \\ J & \text { quanta/(m }{ }^{2} \text { s)) } \\ k(\text { II) } & \text { Sigmoidicity/connectivity parameter } \\ \text { LC } & \text { Rate constant of PS II turnover (1/ms) } \\ \text { LED } & \text { Light curve of fluorescence parameters } \\ \text { MF } & \text { Light-emitting diode } \\ \text { ML } & \text { Frequency of pulse-modulation } \\ M T & \text { Pulse-modulated measuring light } \\ \text { NPQ } & \text { Multiple-turnover light pulse } \\ \text { OEC } & \text { Non-photochemical quenching } \\ & \text { Oxygen-evolving complex }\end{array}$




\begin{tabular}{|c|c|}
\hline$O-I_{1}$ & Photochemical phase of fast fluorescence rise \\
\hline PAM & Pulse amplitude modulation \\
\hline PAR & $\begin{array}{l}\text { Photosynthetically active radiation ( } \mu \mathrm{mol} \\
\text { quanta } /\left(\mathrm{m}^{2} \mathrm{~s}\right) \text { ) }\end{array}$ \\
\hline PAR(II) & $\begin{array}{l}\text { Absolute rate of PS II turnover, quanta/ } \\
\text { (PS II s) }\end{array}$ \\
\hline PPFR & $\begin{array}{l}\text { Photosynthetic photon fluence rate ( } \mu \mathrm{mol} \\
\text { quanta/ }\left(\mathrm{m}^{2} \mathrm{~s}\right) \text { ) }\end{array}$ \\
\hline $\operatorname{Sigma}(\mathrm{II})_{\lambda}$ & $\begin{array}{l}\text { Wavelength-dependent cross section of PS II } \\
\left(\mathrm{nm}^{2}\right)\end{array}$ \\
\hline SP & Saturation pulse \\
\hline ST & Single-turnover light pulse \\
\hline Tau & $\begin{array}{l}\text { Time constant of light-driven } \mathrm{Q}_{\mathrm{A}} \text {-reduction } \\
(\mathrm{ms})\end{array}$ \\
\hline Tau(reox) & Time constant of $\mathrm{Q}_{\mathrm{A}}$ reoxidation $(\mathrm{ms})$ \\
\hline (II) & Effective quantum yield of PS II \\
\hline$\sigma_{\text {PSII }}$ & $\begin{array}{l}\text { Functional absorption cross section of PS II } \\
\left(\mathrm{nm}^{2}\right)\end{array}$ \\
\hline
\end{tabular}

\section{Introduction and instrument methodology}

Since its introduction, now more than 25 years ago (Schreiber et al. 1986), pulse amplitude modulation (PAM) fluorometry in conjunction with the saturation pulse (SP) method has become a routine tool for non-invasive assessment of photosynthetic electron transport in higher plants, algae, and cyanobacteria (Schreiber 2004; reviews in Papageorgiou and Govindjee 2004). In particular, PAMmeasurements of maximal and effective PS II quantum yields via the fluorescence parameters $F_{\mathrm{v}} / F_{\mathrm{m}}=\left(F_{\mathrm{m}}-F_{\mathrm{o}}\right) /$ $F_{\mathrm{m}}$ and $\mathrm{Y}(\mathrm{II})=\left(F_{\mathrm{m}}^{\prime}-F\right) / F_{\mathrm{m}}^{\prime}$ (Genty et al. 1989) have proven of considerable practical relevance. In most applications, relative changes of these parameters are of primary interest, e.g., caused by photoinhibition or other types of environmental stress. The same is true for the ETR parameter, derived from Y(II), which provides a relative measure of linear electron transport rate (Schreiber et al. 1994). Determination of absolute values of $F_{\mathrm{v}} / F_{\mathrm{m}}, \mathrm{Y}(\mathrm{II})$ and ETR is complicated by non-PS II fluorescence (e.g., originating in PS I or in the phycobilisomes) and by the difficulty to determine the quantum flux density (or photon fluence rate) of PS II-absorbed actinic light (AL), which depends on chlorophyll content and the PS II absorption spectrum as well as on the color of the applied light. Information on PS II absorption is a prerequisite for assessment of photosynthetic electron transport rates in optically thin suspensions of algae and cyanobacteria via chlorophyll fluorescence measurements, which is particularly true for estimation of primary productivity by phytoplankton in natural waters. Hence, it is not surprising that the methodology for determination of PS II-specific light absorption and assessment of absolute ETR values has been particularly advanced by researchers in oceanography and limnology (Falkowski and Raven 2007; Kolber et al. 1998). In the study of leaves, which absorb almost all incident photosynthetically active radiation (PAR) most researchers simply have been assuming that $84 \%$ of incident PAR is absorbed (Björkman and Demmig 1987), being evenly distributed between PS I and PS II. This approach has been justified by satisfactory agreement with simultaneous measurements of the rate of $\mathrm{CO}_{2}$ fixation (Genty et al. 1989; Krall and Edwards 1990; Siebke et al. 1997).

While determination of PS II absorption in leaves is complicated by wavelength-dependent intra-leaf light gradients (Vogelmann 1993), it can be realized in a straight forward way in optically thin suspensions via chlorophyll fluorescence measurements. Ley and Mauzerall (1982) introduced the term of the functional absorption cross section of PS II, $\sigma_{\mathrm{PSII}}$, which is measured via the flashintensity saturation curve of the fluorescence increase induced by single-turnover (ST) flashes. This approach has been applied extensively and further developed by Falkowski and co-workers (Falkowski and Kolber 1995; Falkowski et al. 2004; Falkowski and Raven 2007; Kolber et al. 1998). The development from the original pump-andprobe method toward fast repetition rate (FRR) fluorometry has been converging with parallel developments in PAM fluorometry (Jakob et al. 2005; Kolbowski and Schreiber 1995; Neubauer and Schreiber 1987; Schreiber 1986; Schreiber et al. 1993, 1995, 2011). With current instrumentation, both approaches allow measurements of the fluorescence rise induced by strong $\mathrm{AL}$, estimation of the functional absorption cross section of PS II and assessment of maximal and effective PS II quantum yields after singleor multiple-turnover (MT) closure of the PS II acceptor side.

In contrast to leaves, which show relatively flat absorption spectra, dilute suspensions of unicellular algae and cyanobacteria display pronounced wavelength-dependent differences of PS II absorption, which are reflected in characteristic fluorescence excitation spectra, representing the "finger-prints" of the various types of PS II antenna pigment-systems (cyanobacteria, cryptophytes, green algae, diatoms/dinoflagellates). Multi-wavelength PAM fluorometers have been developed to estimate the content of various pigment-groups of phytoplankton in mixed natural waters (Beutler et al. 2002; Kolbowski and Schreiber 1995), by deconvolution of the overall signal into several components, based on "reference spectra" for the major pigmentgroups. However, as was pointed out by Jakob et al. (2005), reliability and accuracy of this approach are limited by potential differences between $\sigma_{\mathrm{PSII}}$ of the various types of 
phytoplankton in natural waters and the laboratory-grown cultures used for measurements of "reference spectra". Furthermore, not only the differences in $\sigma_{\text {PSII }}$ between the various types and adaptation states of phytoplankton have to be considered but also the wavelength dependence of $\sigma_{\text {PSII }}$.

While the theory of FRR fluorometry (Kolber et al. 1998) in principle does account for species and wavelength dependence of $\sigma_{\text {PSII }}$, in practice, in situ measurements normally are carried out with naturally occurring mixed samples and a single color of measuring and AL, so that the obtained parameters $F_{\mathrm{v}} / F_{\mathrm{m}}$ and $\sigma_{\text {PSII }}$ cannot give specific information. Hence, relative changes in these parameters can be interpreted only if changes in relative contents of different pigment types can be excluded. In most FRR studies, blue light has been used, as this approximates the spectral light quality in marine environments, the PS II absorption of which differs considerably between different types of phytoplankton. This aspect is dealt with in a recent report on FRR measurements by Suggett et al. (2009) who state: "It is now becoming clearer that in situ values of Fv/ Fm and $\sigma_{\text {PSII }}$ also contain taxonomic information" and "The magnitudes of variability in Fv/Fm and $\sigma_{\text {PSII }}$ driven by changes in phytoplankton community structure often exceed that induced by nutrient limitation."

Most PAM fluorometers just provide one color of pulsemodulated measuring light (ML) (normally red or blue), with the option of applying AL of any spectral composition, including natural sun light. With the XE-PAM (Schreiber et al. 1993), which employs xenon-discharge flashes for both ML and saturating ST flashes, the colors of measuring and AL can be defined with the help of optical filters. While this instrument allows estimation of $\sigma_{\text {PSII }}$ by the pump-and-probe method, this approach has not been much used, as it is time-consuming and requiring considerable background knowledge and experimental skill. The phyto-PAM (Jakob et al. 2005; Kolbowski and Schreiber 1995) employs four different colors for ML, but just one color of AL (red) and, hence, does not allow estimating the wavelength-dependent $\sigma_{\text {PSII }}$. The microfiber-PAM (Schreiber et al. 1996) offers four different colors for measuring and AL. This device, however, lacks the time resolution for assessment of rapid rise kinetics, required to estimate $\sigma_{\mathrm{PSII}}$. The same is also true for a recently introduced multi-color PAM fluorescence imaging system (Trampe et al. 2011). Finally, the very recently developed multi-color-PAM (Schreiber et al. 2011) provides six different colors of ML and six different colors of AL, all of which qualify for highly accurate measurements of fast induction kinetics and assessment of wavelength-dependent $F_{\mathrm{v}} / F_{\mathrm{m}}$ and functional absorption cross section of PS II. This new device is the topic of the present communication.

In practice, for correct determination of the wavelengthdependent $\sigma_{\text {PSII }}$ via the fluorescence rise kinetics in strong light, a number of physiological factors have to be considered, which affect the rise kinetics and, hence, had to be accounted for in the development of the methodology: (1) state of light acclimation of the sample (state 1 or state 2); (2) redox state of the PS II acceptor side, including the PQpool; (3) limitation of PS II turnover at very high light intensities (fluorescence rise within about $100 \mu \mathrm{s}$ ) by a non-photochemical loss process (Rappaport et al. 2007); (4) quenching of fluorescence at the so-called $I_{1}$-level (Samson et al. 1999; Schreiber 1986, 2004; Schreiber and Krieger 1996). Consideration of these factors has led to a somewhat modified approach for determination of the functional absorption cross section of PS II, with respect to the pump-and-probe and FRR methods. The measurement is carried out with the sample being in a defined quasi-dark (+far-red, FR)-adapted "reference state" using relatively moderate actinic intensities (fluorescence rise within about $1 \mathrm{~ms}$ ), with maximal fluorescence yield (i.e., $I_{1}$-level at saturation of photochemical phase) being induced at the end of the rise curve by a saturating ST flash. Therefore, the functional PS II absorption cross section measured with the multi-color-PAM is valid only for the reference state in which it was measured and any changes of PS II efficiency occurring, e.g., during illumination are assumed to be covered by corresponding changes in the effective PS II quantum yield, Y(II). For this reason, to avoid confusion with the previously defined $\sigma_{\text {PSII }}$, which changes during illumination and in response to chlororespiratory electron flow (Koblizek et al. 2001), the wavelength-dependent functional PS II absorption cross section determined with the multi-color-PAM is called $\operatorname{Sigma}(\mathrm{II}){ }_{\lambda}$.

For correct assessment of $\operatorname{Sigma}(\mathrm{II})_{\lambda}$, it is essential that the quantum flux density of the incident PAR is homogeneous, which can be realized only at rather low chlorophyll content (below about $500 \mu \mathrm{g} \mathrm{Chl/L} \mathrm{in} \mathrm{suspensions),} \mathrm{thus}$ excluding straight forward measurements with leaves. However, even with optically dense objects valuable information can be obtained by application of different colors of light, differing in depths of penetration, a topic that recently has received considerable attention (Oguchi et al. 2011; Rappaport et al. 2007; Takahashi et al. 2010; Terashima et al. 2009), with the first and the two latter studies concentrating on the wavelength dependence of photoinhibition.

There has been general agreement that PS II is the primary target of photoinhibition and can be measured via the decrease in $F_{\mathrm{v}} / F_{\mathrm{m}}$ (Demmig-Adams and Adams 1992). The molecular mechanism of the primary photodamaging reaction, however, is still controversial. Recently, the socalled two-step hypothesis has been advanced (Hakala et al. 2005; Nishiyama et al. 2006; Ohnishi et al. 2005), which suggests that the primary step involves damage of the oxygen-evolving complex (OEC), when the Mn-cluster 
dissociates after absorption of UV or blue light; donor-side limitation of PS II is supposed to induce secondary damage upon light absorption of PS II pigments, due to the increased life time of $\mathrm{P} 680^{+}$and the resulting formation of singlet oxygen. This hypothesis is supported by action spectra of photodamage to PS II with peaks in the UV-A and blue region, resembling those of model manganese compounds and differing considerably from PS II absorption spectra (Hakala et al. 2005).

Whereas measurements of the wavelength dependence of photoinhibition in leaves are complicated by intra-leaf light gradients and fluorescence reabsorption, it can be investigated in a straight forward way in optically thin suspensions. As this topic is close to the heart of Osmond (1981, 1994) to whom this contribution is dedicated, in addition to the technical and methodological aspects of the multi-color-PAM also an application of this new device in the study of the wavelength dependence of photoinhibition will be presented. In this application, use of the possibility is made to adjust defined rates of quanta absorption by PS II with blue and red lights in a dilute suspension of Chlorella. If photoinhibition were just an unavoidable consequence of PS II turnover, equal turnover rates should induce equal loss in PS II quantum yield. It will be shown that the damaging effect is distinctly larger with blue light.

\section{Materials and methods}

\section{Experimental setup}

The experiments were carried out with a first prototype of a multi-color-PAM chlorophyll fluorometer developed by the authors, which recently has become commercially available (Heinz Walz GmbH, Germany). This device is based on a chip on board (COB) light-emitting diode (LED) array consisting of 60 Power-LED chips mounted on a $10 \times 10 \mathrm{~mm}$ area, featuring a total of eight different colors, which serve for pulse-modulated ML, AL, FR light, ST pulses, and MT pulses, equivalent to SP. Figure 1 shows a block diagram of the experimental setup. The emitterdetector units are mounted on an Optical Unit with four light-ports (ED-101US/MD), essentially identical to the one introduced for the XE-PAM and phyto-PAM chlorophyll fluorometers (Kolbowski and Schreiber 1995; Schreiber et al. 1993).

Light emission by the multi-color LED array (1) is controlled by separate LED drivers for the various light qualities, which are triggered with $2.5-\mu$ s time resolution under firmware/software control. The light passes a shortpass dichroic filter $(<640 \mathrm{~nm})$ (2) before it enters a $10 \times 10 \mathrm{~mm}$ Perspex rod (3) that guides it to the $10 \times 10 \mathrm{~mm}$ glass cuvette (4), mixing the various light

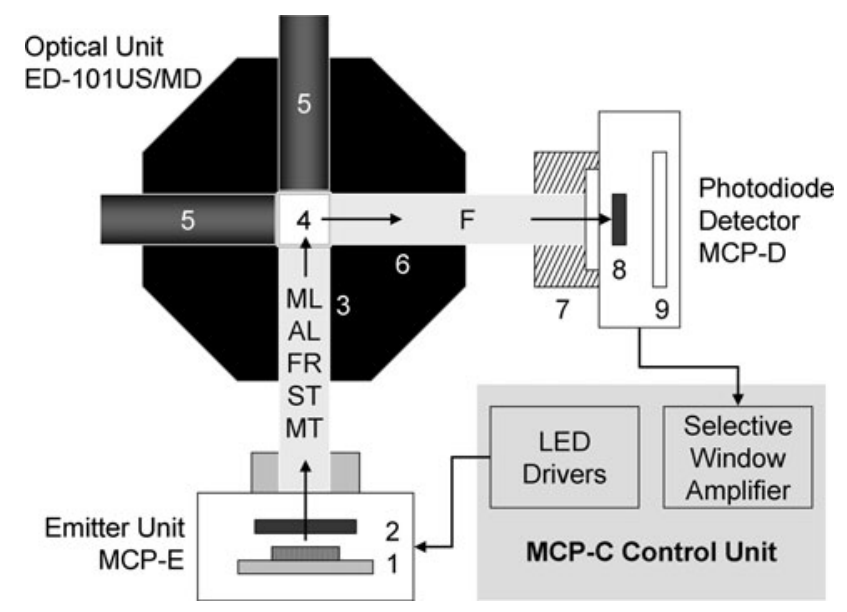

Fig. 1 Block diagram of the multi-color-PAM set-up for measurements with suspensions using the optical unit ED-101US/MD (see text for explanations)

qualities by multiple reflections. The suspension within the cuvette (4) is continuously stirred with the help of a small magnetic "flea." Push-in rods with mirror front faces (5) are inserted at $90^{\circ}$ and $180^{\circ}$ angles to incident light, thus increasing both the effective light intensities and the amount of fluorescence picked up by a $10 \times 10 \mathrm{~mm} \mathrm{Per-}$ spex rod (6) at $90^{\circ}$ angle to incident light. The fluorescence $(F)$ passes a long-pass glass-filter $(>650 \mathrm{~nm}$, normally $3 \mathrm{~mm}$ RG665) (7), which absorbs scattered incident light, so that only fluorescence reaches the $10 \times 10 \mathrm{~mm}$ photodiode detector (8). The pulse-modulated fluorescence signal selectively is amplified by a pulse-preamplifier (9) within the detector-unit and then further processed by a special selective-window amplifier within the main control unit.

For standard fluorescence measurements, pulse-modulated ML with peak-wavelengths at 440, 480, 540, 590, and $625 \mathrm{~nm}$ is provided (for special applications, not dealt with in this communication, also 400 or $365 \mathrm{~nm}$ ML is available). ML pulses, displaying a width of $1 \mu$ s, can be applied at wide ranges of pulse intensities (20 settings) and frequencies $(10-100,000 \mathrm{~Hz})$, so that time-integrated intensities may differ by a factor of $2 \times 10^{5}$, reaching from virtual darkness to almost saturating light (depending on color and investigated organism).

A separate set of otherwise identical LED-chips with peak-wavelengths at 440, 480,540,590, and $625 \mathrm{~nm}$ serves for actinic illumination (AL, ST, MT, or SP), supplemented with a white Power-LED (420-645 nm). The latter particularly contributes to saturating multi-color ST. In addition, for preferential excitation of photosystem I (PS I), the LED array features a $725 \mathrm{~nm}$ (FR) Power-LED, which is mounted such that the FR can enter the Perspex rod (3) without being blocked by the short-pass filter (2). 
ST pulses can be applied either with single colors (normally non-saturating) or all colors simultaneously (generally saturating). The "ST pulse intensity," is adjusted via the width that can be set between 2.5 and $50 \mu$ s. Pulse current is always maximal for ST pulses. In contrast, MT pulses or SPs can be applied using single colors only, with the intensity being adjusted via pulse currents (20 settings). While MT pulses and SPs, employing the same LED drivers, optically are fully equivalent, they serve different functions. MT pulses can be triggered with $2.5-\mu \mathrm{s}$ resolution by preprogrammed Fast Trigger files (possible widths ranging from $2.5 \mu \mathrm{s}$ to $800 \mathrm{~ms}$ ) for measurements of fast induction or relaxation kinetics. On the other hand, SP specifically serve for determination of $F_{\mathrm{m}}$ and $F_{\mathrm{m}}^{\prime}$ in SP quenching analysis (see van Kooten and Snel 1990; Schreiber 2004 for nomenclature). Different SP intensities can be set for $F_{\mathrm{m}}$ and $F_{\mathrm{m}}^{\prime}$ determination (default settings 3 and 10 , respectively), as distinctly less intensity is required to saturate the PS II acceptor side after dark-adaptation than in the illuminated state, when the PS I acceptor side is light activated.

\section{Measurements of fluorescence yield}

The fluorescence yield of a dark-adapted sample, $F_{\mathrm{o}}$, generally is measured using low frequency $(10-50 \mathrm{~Hz})$ of pulse-modulated ML corresponding to photon fluence rates $\ll 1 \mu \mathrm{mol} /\left(\mathrm{m}^{2} \mathrm{~s}\right)$, so that no accumulation of reduced photosystem II (PS II) acceptors can occur. In principle, the integrated intensity of the ML can be sufficiently low (at still satisfactory signal/noise ratio) that closure of so-called inactive PS II (Lavergne and Leci 1993) is avoided. In most experiments, however, FR background light is applied to establish reproducible control conditions in terms of an oxidized plastoquinone (PQ) pool and state 1 (Mullineaux and Emlyn-Jones 2005). FR preillumination results in a rapid small fluorescence increase (about $10 \%$ of $F_{\mathrm{o}}$ ) due to the response of "inactive PS II" and a more or less pronounced slow rise of $F_{\mathrm{o}}\left(t_{1 / 2}\right.$ in the order of $\left.5 \mathrm{~min}\right)$ reflecting a state 2 -state 1 shift (depending on type of cells, temperature, etc.).

The fluorescence yield of an illuminated sample, $F$, normally is measured at substantially higher frequency of pulse-modulated ML (measuring light frequency, MF, 1$100 \mathrm{kHz}$ ) than in the case of $F_{\mathrm{o}}$, with correspondingly enhanced signal/noise ratio and time resolution. Consequently, ML normally contributes significantly to overall actinic intensity, which is accounted for in the PAR value indicated by the user software (see below). In the experiments described in this communication, photons of ML and AL/MT/ST are fully equivalent, as the same colors (batches of LED-chips) were used for all of them.
Slow changes of fluorescence yield were measured in the SP-analysis mode of the software program (PamWin-3). Fluorescence yields $F_{\mathrm{m}}$ and $F_{\mathrm{m}}^{\prime}$ were measured with $300 \mathrm{~ms}$ SP width. Based on the measured values of $F_{\mathrm{o}}, F_{\mathrm{m}}, F$, and $F_{\mathrm{m}}^{\prime}$ the PamWin-3 program automatically calculates maximal and effective PS II quantum yields, $F_{\mathrm{v}} / F_{\mathrm{m}}$, and $\mathrm{Y}(\mathrm{II})$, respectively, as well as various other derived fluorescence parameters (Klughammer and Schreiber 2008; Kramer et al. 2004; van Kooten and Snel 1990).

Light response curves (LC) of relative ETR (rel.ETR) were recorded with the help of Light Curve Program files (lcp-files) programmed for the different colors of light. In general, the same colors were used for ML and AL. Step width at each intensity setting was $3 \mathrm{~min}$. The low-intensity steps were covered by ML at high settings of pulsefrequency. Before start of the LC, samples were darkadapted for $30 \mathrm{~min}$ in the presence of weak FR background light (minimal setting 1) and $O-I_{1}$ rise curves were recorded for assessment of Sigma(II $)_{\lambda}$, the absorption cross section of PS II (see below).

Dark-light-dark induction/recovery curves were measured under the control of Script-file programmed for this purpose. With the help of Script-files, practically all commands that can be carried out manually, can also be programmed with defined time steps between consecutive commands, for fully automated recording. In this way, the experiments were carried out with high reproducibility. Fresh samples were used for each run, which were darkadapted for $15 \mathrm{~min}$ in the presence of weak FR light that was applied throughout the experiment. Identical cell densities were adjusted via identical $F_{\mathrm{o}}$ signals measured with $440 \mathrm{~nm}$ ML at fixed settings of ML-intensity and Gain. When another color of light was used for the actual measurement of light-induced changes, after adjustment of cell densities equal $F_{\mathrm{o}}$ levels were adjusted via the settings of ML-intensity and Gain, with fine adjustment via the distance between cuvette and photodiode detector (see Fig. 1).

Measurement of light intensity and PAR-lists

The photon fluence rate (or quantum flux density) of PAR was measured with a calibrated quantum sensor (US-SQS/ WB, Walz), featuring a 3.7-mm diffusing sphere, mounted in the center of the cuvette filled with water. This sensor is connected via an amplifier box directly to the External Sensor input of the MCP-C Control Unit. The PamWin software provides a routine for automated measurements of ML, AL, and MT/SP intensities of all the colors at 20 settings each. The measured values are saved in the socalled PAR-lists, on which calculation of PAR-dependent parameters is based. PAR and fluorescence measurements 
were carried out under close to identical optical conditions. Detailed knowledge of incident PAR (in units of $\mu \mathrm{mol} /$ $\left.\left(\mathrm{m}^{2} \mathrm{~s}\right)\right)$ effective within the suspension during illumination with different colors of ML, AL, and MT/SP is essential for quantitative analysis of the light responses. As all measurements were carried out at low cell densities, also transmitted light reflected back into the sample (see Fig. 1) contributed significantly to overall intensity, which was accounted for using the spherical sensor. While strictly speaking in this case the term photosynthetic photon fluence rate (PPFR) may apply (Braslavsky 2007), for the sake of simplicity in PAM applications the abbreviation PAR has been used.

Measurements of fast kinetic responses

Fast kinetic responses were measured under the control of so-called Fast Trigger files, which were programmed such that rapid changes of light intensity, as occurring upon ALon/off, MT-on/off, or during an ST pulse, do not affect the pulse-modulated signal. The Sample-and-Hold off ( $\mathrm{S} \& \mathrm{H}$ off) Trigger is essential for avoiding artifacts induced by rapid changes of non-modulated light. During the S\&H off time the sample-and-hold amplifier, which processes the pulse-modulated signal, is "gated" (i.e., switched off). Figure 2 shows a screenshot of the Fast Trigger pattern of the file Sigma1000.FTM that was programmed for reproducible measurements of the so-called $O-I_{1}$ rise kinetics (for nomenclature see Schreiber 2004) and determination of the sample- and wavelength-dependent absorption cross section of PS II, Sigma(II) $\lambda$, which play a central role in the present report.

The Fast Trigger pattern of the file Sigma1000.FTM displayed in Fig. 2 may serve to outline some points essential for optimal measurements of the $O-I_{1}$ rise kinetics:

(1) The pulse-modulated fluorescence ML is switched on only $100 \mu \mathrm{s}$ before onset of AL to minimize the fluorescence rise induced by the ML and, hence, to allow use of relatively high ML-intensity setting for the sake of a high signal/noise ratio.

(2) Maximal measuring pulse-frequency $\left(\mathrm{MF}_{\max }\right)$ is triggered simultaneously with ML-on. The default setting of $\mathrm{MF}_{\max }=100 \mathrm{kHz}$ provides sufficient time resolution for reliable assessment of the $O-I_{1}$ kinetics with time constants in the order of $200 \mu$ s.

(3) $\mathrm{AL}$ is triggered at time $-5 \mu$ s to take account of a small time delay between switching of the AL-LEDdriver and AL-on.

(4) The amplifier "gating" ( $\mathrm{S} \& \mathrm{H}$ off) is triggered on for $15 \mu \mathrm{s}$ for AL-on (from -10 to $5 \mu \mathrm{s}$ ) and for $80 \mu \mathrm{s}$ for the $50 \mu \mathrm{s}$ ST pulse (from 995 to $1,075 \mu \mathrm{s}$ ).

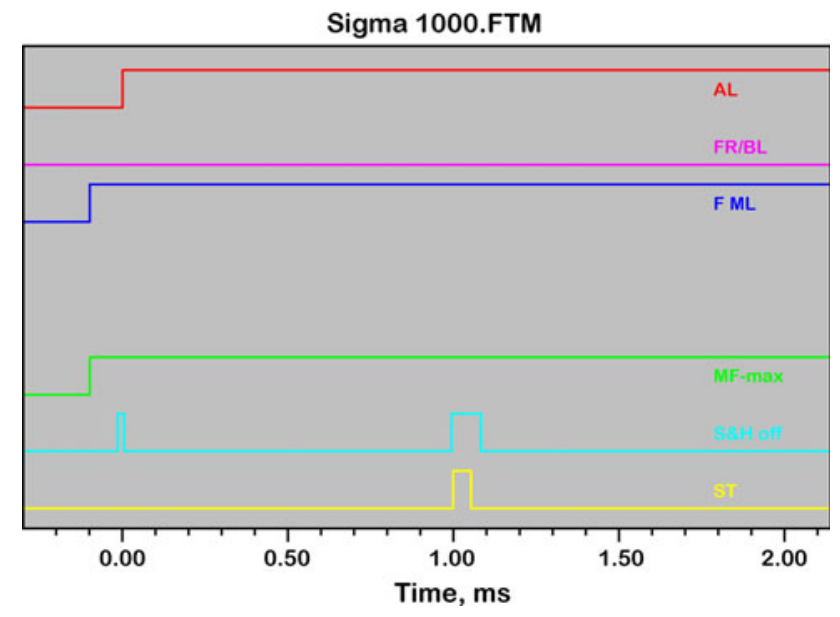

Fig. 2 Screenshot of the Fast Trigger pattern programmed for measurements of $O-I_{1}$ rise kinetics. On-off times of six different triggers are depicted: $A L$ actinic light, $F R / B L$, far-red or blue light pretrigger PS I illumination (not active, as FR background instead), $F$ $M L$ pulse-modulated fluorescence $\mathrm{ML}, M F_{\max }$ maximal ML frequency, 100 or $200 \mathrm{kHz}, S \& H$ off Sample\&Hold off gating period of amplifier, $S T$ single-turnover pulse (see text for further explanations)

Consecutive measurements of $O-I_{1}$ rise kinetics driven by strong 440-, 480-, 540-, 590-, and 625-nm light of the same sample were preprogrammed in special Script-files for Chlorella and Synechocystis with 10-s dark-time between measurements. For each color, ML-intensity/Gain settings were programmed to give approximately equal $F_{\mathrm{o}}$ values. AL/MT-intensity settings were programmed such that for the investigated organism the initial rise curves displayed similar slopes with all the colors.

\section{Analysis of $O-I_{1}$ rise kinetics}

The kinetics of the $O-I_{1}$ fluorescence rise were analyzed with the help of a dedicated fitting routine developed for determination of the wavelength-dependent absorption cross section of PS II, here called Sigma(II) $)_{\lambda}$. Fitting is based on the reversible radical pair model of PS II originally described by Lavergne and Trissl (1995) that was extended to take account of $\mathrm{Q}_{\mathrm{A}}^{-}$-reoxidation (Klughammer $\mathrm{C}$, Kolbowski J and Schreiber U, in preparation). Variable parameters in this model, fitted by the PamWin-3 program, are:

$J \quad$ Sigmoidicity parameter, which is related to Joliot's connectivity parameter, $p$, via the equation $J=p /(1-p)$

Tau Time constant of light-driven reduction of $\mathrm{Q}_{\mathrm{A}}$ (by AL or MT pulse), corresponding to the inverse of the rate constant of PS II turnover, $k(\mathrm{II})$ 
Tau(reox) Time constant of $\mathrm{Q}_{\mathrm{A}}^{-}$-reoxidation.

Directly measured parameters are the $F_{\mathrm{o}}$ and $I_{1}$-levels, which define the total range of $\Delta F$ that can be induced by a saturating ST flash (ST pulse) in the presence of an oxidized PQ-pool. The fitted parameters refer to the kinetics of $\mathrm{Q}_{\mathrm{A}}$-reduction, i.e., the increase of $(1-q)$, where $q$ represents the fraction of open PS II reaction centers. The relationship between variable fluorescence and $(1-q)$ is described by the equation $\left(F-F_{\mathrm{o}}\right) /\left(I_{1}-F_{\mathrm{o}}\right)=(1-q) /$ $(1+J q)$ in analogy to an equation derived by Lavergne and Trissl (1995).

The $O-I_{1}$ curves measured with the five different colors were fitted together with the restriction of common values of $J$ and Tau(reox), as these parameters are unlikely to depend on the color of light. Calculation of $\operatorname{Sigma}(\mathrm{II})_{\lambda}$ by the multi-color-PAM-software is based on the fitted value of the time constant Tau and the value of incident PAR, using the following general equation:

$\operatorname{Sigma}(\mathrm{II})_{\lambda}=\frac{k(\mathrm{II})}{L \cdot \mathrm{PAR}}=\frac{1}{\tau \cdot L \cdot \mathrm{PAR}}$,

where $k(\mathrm{II})$ is the rate constant of PS II turnover and Tau the time constant of $\mathrm{Q}_{\mathrm{A}}$-reduction during the $O-I_{1}$ rise, $L$ is Avogadro's constant, PAR is the photon fluence rate of the light driving the $O-I_{1}$ rise and Sigma(II) $)_{\lambda}$ the wavelengthand sample-dependent absorption cross section of PS II (for further explanations, see "Results and interpretation" section).

\section{Measurement of absorptance}

Sample absorptance was measured using the same Optical Unit ED-101US/MD as for fluorescence measurements (see Fig. 1), but with the detector-unit MCP-D being moved from the $90^{\circ}$ position (relative to the emitter-unit) to the $180^{\circ}$ position. The long-pass filter in front of the detector was exchanged against suitable neutral density filters and pin-hole diaphragms, so that pulse-modulated transmittance signals could be measured both with the suspension medium as such, $I_{\text {medium }}$, and with the suspension medium containing Chlorella or Synechocystis, $I_{\text {sample }}$. The absorptance a (=1 - transmittance) was calculated as $a=1-I_{\text {sample }} / I_{\text {medium. }}$. With the given optical geometry almost all light entering the $10 \times 10 \mathrm{~mm}$ cuvette via the emitter-perspex-rod is picked up by the detector-perspexrod, unless absorbed by the sample.

Photosynthetic organisms and sample preparation

Experiments were carried out with dilute suspensions of green unicellular algae Chlorella vulgaris and cyanobacteria Synechocystis PCC 6803. Chlorella was cultured in natural day light (north window) at 20-40 $\mu \mathrm{mol} /$ $\left(\mathrm{m}^{2} \mathrm{~s}\right)$ and room temperature $\left(25^{\circ} \mathrm{C}\right)$ in an inorganic medium (Pirson and Ruppel 1962) under ambient air. Synechocystis was grown photoautotrophically in artificial light (tungsten) at $30 \mu \mathrm{mol} /\left(\mathrm{m}^{2} \mathrm{~s}\right)$ and $30{ }^{\circ} \mathrm{C}$ in Allen's (1968) medium under ambient air. Both cultures were shaken manually at least four times per day. Cultures were frequently diluted so that chlorophyll content did not exceed 5-10 mg/L. Experiments were carried out at room temperature with diluted suspensions at $200-300 \mu \mathrm{g} / \mathrm{L}$, as determined with a calibrated WATER-PAM chlorophyll fluorometer (Walz).

For sample preparation the cuvette was first filled with $1.4 \mathrm{~mL}$ of culture medium and then stock suspension was added dropwise to the stirred sample until signals corresponding to $200-300 \mu \mathrm{g} / \mathrm{L}$ were reached. Settings of MLintensity and Gain were adjusted to obtain $F_{\mathrm{o}}$ values of about 2 and $3 \mathrm{~V}$ in the case of Chlorella and Synechocystis, respectively.

\section{Results and interpretation}

Wavelength dependence of normalized $F_{\mathrm{o}} / \mathrm{PAR}$ and absorptance

The most important parameters determining the intensity of chlorophyll fluorescence are (1) quantum flux density of incident photosynthetically active light (PAR), (2) spectral composition of the incident light, (3) absorption spectrum of the photosynthetic organism, (4) cell density/chlorophyll content and (5) state of PS II in terms of reduction of the primary acceptor $\mathrm{Q}_{\mathrm{A}}$ and down-regulation by non-photochemical quenching (NPQ). The effect of the last parameter can be considered constant, when samples are darkacclimated in the presence of weak FR light that oxidizes the PQ-pool resulting in the so-called state 1, provided the intensity of the pulse-modulated ML is sufficiently low, so that it does not change the state of PS II. When this prerequisite is fulfilled, at constant PAR of incident $\mathrm{ML}$ and chlorophyll content of the sample, the wavelength dependence of the fluorescence signal reflects the overlapping integral between the spectrum of the incident light and the absorption spectrum of the photosynthetic pigments that transfer the excitation energy to PS II. When narrow band excitation is used, as is the case with standard spectrofluorometers, fluorescence intensity per incident quanta measured as a function of wavelength results in an excitation spectrum. The multi-color-PAM provides relatively broad-band light (half-band width 15-25 nm) peaking at $440,480,540,590$, and $625 \mathrm{~nm}$, resulting in a coarse fivepoint excitation spectrum. 
In Fig. $3 \mathrm{~A}$ and Table 1 , the $F_{\mathrm{o}}$ values measured with 440, 480, 540, 590, and $625 \mathrm{~nm}$ ML in dilute suspensions of green algae (Chlorella vulgaris) and cyanobacteria (Synechocystis PCC 6403) are compared using identical settings of Gain (signal amplification). The cell densities in the two suspensions were adjusted to give the same absorptance at $440 \mathrm{~nm}$ (see "Materials and methods"). At the applied ML-intensity settings the intensities of the incident PAR generally were too low to induce any fluorescence increase beyond $F_{\mathrm{O}}$ (even with respect to "inactive PS II"). Division of the measured $F_{\mathrm{O}}$ values by the incident PAR (derived from instrument specific PAR-lists) and normalization results in the so-called PAR-scaled $F_{\mathrm{o}}$ values, equivalent to $F_{\mathrm{o}}$ values as would be measured with equal photon fluence rates at different wavelengths. PARscaled $F_{\text {o }}$ plotted against the peak-wavelengths corresponds to a fluorescence excitation spectrum (see Fig. 3A). The $F_{\mathrm{o}} / \mathrm{PAR}$ data were normalized to 1 relative unit at the maximal signal value, which was observed with Synechocystis using 625-nm excitation.

As may be expected in view of the differences in photosynthetic pigments serving PS II, the wavelength dependence of dark-fluorescence yield, $F_{\mathrm{o}}$, differs considerably between Chlorella and Synechocystis. Somewhat unexpectedly, despite the identical absorptance at $440 \mathrm{~nm}$, i.e., although the same fraction of incident $440 \mathrm{~nm}$ quanta is absorbed in the Chlorella and Synechocystis suspensions, the $F_{\mathrm{o}}(\text { Chlorella })_{440}$ exceeds the $F_{\mathrm{o}}(\text { Synechocystis })_{440}$ by a factor of 2.294/0.359 = 6.4 (see Table 1). Absorption at $440 \mathrm{~nm}$ is dominated by Chl $a$ and, hence, Chl $a$ concentration should be close to identical in the two samples. The large difference in $F_{\mathrm{o}} / \mathrm{PAR}$ values may be explained by a higher fluorescence yield of Chl $a$ (PS II) as compared to Chl $a$ (PS I) and to a higher PS I/PS II ratio in Synechocystis than in Chlorella. In contrast, when with the same samples $625 \mathrm{~nm}$ ML is used, the $F_{\mathrm{o}}$ (Synechocystis) ${ }_{625}$ exceeds the $F_{\mathrm{o}}(\text { Chlorella })_{625}$ by a factor of $1.702 /$ $0.522=3.3$. In Synechocystis, the peak of absorption by phycocyanin is at $625 \mathrm{~nm}$, whereas in Chlorella this wavelength is at some distance from the main Chl al $b$ absorption peaks.

The $F_{\mathrm{O}} / \mathrm{PAR}$ plots of Chlorella and Synechocystis in Fig. 3A can be compared with the corresponding absorptance spectra in Fig. 3B, measured under identical optical conditions (see "Materials and methods"). While the spectra of $F_{\mathrm{o}} / \mathrm{PAR}$ and absorptance resemble each other with Chlorella, they differ substantially in the case of Synechocystis. PS I-specific absorption is higher in Synechocystis than in Chlorella due to a higher PS I/PS II ratio. Also, the more PS I-specific absorption differs from PS II-specific absorption, the more the overall absorptance spectrum will differ from the $F_{\mathrm{o}} / \mathrm{PAR}$ spectrum. Therefore,

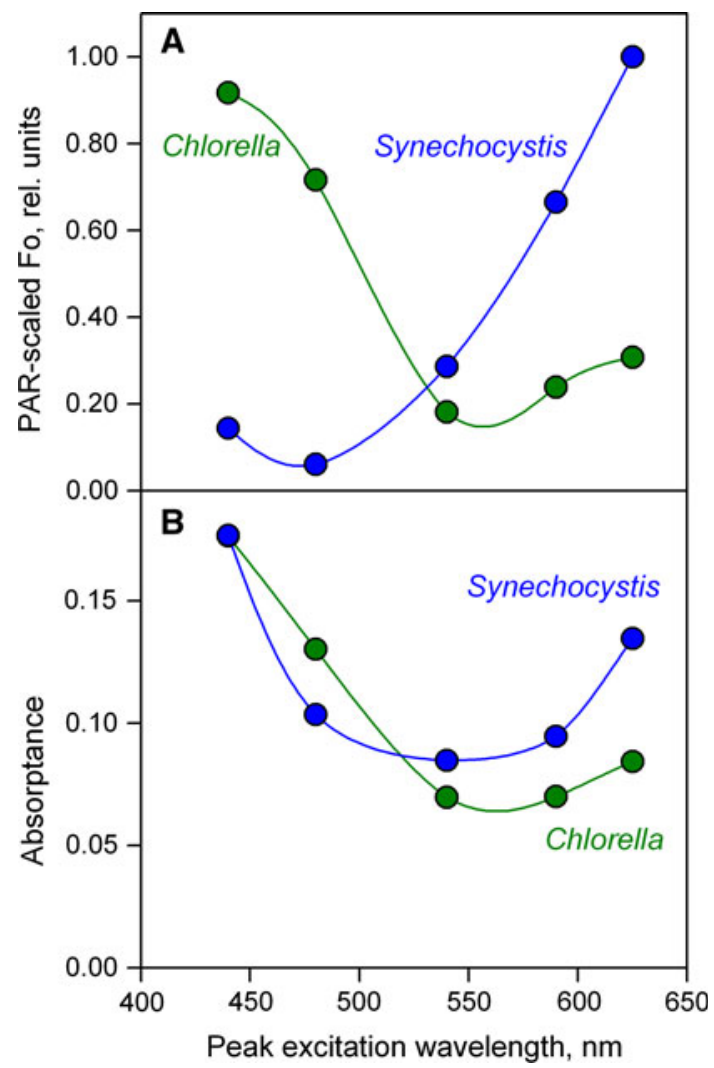

Fig. 3 Comparison of PAR-scaled $F_{\mathrm{o}}$ and absorptance in dilute suspensions of Chlorella and Synechocystis as a function of the color of the pulse-modulated ML. Cell densities of the two suspensions were adjusted to give the same absorptance at $440 \mathrm{~nm}$. A Normalized $F_{\mathrm{o}} / \mathrm{PAR}$ versus peak wavelength of the ML. The data were normalized to unity at maximal relative $F_{\mathrm{o}} / \mathrm{PAR}$, i.e., for $625 \mathrm{~nm}$ with Synechocystis. B Absorptance in the same suspensions plotted vs peak wavelength of the ML

$F_{\mathrm{o}} / \mathrm{PAR}$ spectra can provide more specific information on PS II absorption, than absorptance spectra.

This finding appears important in connection with attempts to model primary production on the basis of measurements of chlorophyll fluorescence and calculation of photosynthetic absorbed radiation $Q_{\text {phar }}$ from surface irradiance and Chl $a$-specific absorption in successive water layers (Gilbert et al. 2000a, b; Jakob et al. 2005). Obviously, the wavelength dependencies of $Q_{\mathrm{phar}}$ and of the rate of PS II-specific quanta absorption can differ substantially. PS II charge-separation rate is decisive for the overall rate of photosynthetic electron transport.

While PAR-scaled $F_{\mathrm{o}}$ may qualify as a satisfactory proxy for estimating the relative extent of PS II excitation by the five different colors of light provided by the multicolor-PAM, it does not carry information on the absolute rates. As will be shown below, such information can be derived from measurements of the wavelength-dependent $O-I_{1}$ rise kinetics. 
Table 1 Comparison of $F_{\mathrm{o}}$ and $F_{\mathrm{o}} / \mathrm{PAR}$ of dilute suspensions of Chlorella and Synechocystis measured with five different colors at identical settings of ML-intensity and minimal pulse-frequency

\begin{tabular}{|c|c|c|c|c|c|}
\hline \multicolumn{6}{|l|}{ Parameter } \\
\hline Peak wavelength (nm) & 440 & 480 & 540 & 590 & 625 \\
\hline $\begin{array}{l}\text { Incident PAR }(\mu \mathrm{mol} / \\
\left.\left(\mathrm{m}^{2} \mathrm{~s}\right)\right)\end{array}$ & 0.0234 & 0.0309 & 0.0201 & 0.0099 & $0.015 \mathrm{C}$ \\
\hline $\begin{array}{l}\text { Incident PAR (rel. } \\
\text { units) }\end{array}$ & 75.7 & 100.0 & 65.2 & 32.0 & 51.5 \\
\hline$F_{\mathrm{o}}(\text { Chlorella })_{\lambda}(\mathrm{V})$ & 2.294 & 2.366 & 0.389 & 0.252 & 0.522 \\
\hline $\begin{array}{l}F_{\mathrm{o}}(\text { Chlorella })_{\lambda} / \mathrm{PAR} \\
\text { (rel. units) }\end{array}$ & 0.917 & 0.716 & 0.181 & 0.238 & 0.307 \\
\hline$F_{\mathrm{o}}(\text { Synechocystis })_{\lambda}(\mathrm{V})$ & 0.359 & 0.198 & 0.616 & 0.703 & 1.702 \\
\hline $\begin{array}{l}F_{\mathrm{o}}(\text { Synechocystis })_{\lambda} l \\
\text { PAR (rel. units) }\end{array}$ & 0.143 & 0.060 & 0.286 & 0.665 & 1.000 \\
\hline
\end{tabular}

The $F_{\mathrm{o}} / \mathrm{PAR}$ values were normalized to give 1 rel. unit at $625 \mathrm{~nm}$ with Synechocystis, where the maximal signal was obtained

Wavelength dependence of relative electron transport rate in Chlorella

The light response of photosynthetic organisms can be routinely analyzed with the help of fluorescence-based light curves (LCs), consisting of a number of illumination steps using increasing intensities of PAR. The longer the illumination steps the more the fluorescence-based LCs approach classical $P-I$ curves (photosynthesis vs. irradiance curves), where steady state is reached within each PAR-step, before photosynthetic rate is evaluated. PAM fluorometers allow more or less rapid LC-recordings of various fluorescencederived parameters, like the effective PS II quantum yield, Y(II), and relative electron transport rate, rel.ETR (see, e.g., Herlory et al. 2007; Ralph and Gademann 2005; Rascher et al. 2000; Schreiber et al. 1994). For LCs with illumination times too short to reach steady state, the term rapid LCs (RLCs) was coined (Schreiber et al. 1997).

Rel.ETR as a fluorescence-derived parameter originally was introduced for PAM-measurements with leaves (Schreiber et al. 1994)

rel.ETR $=\mathrm{Y}(\mathrm{II}) \cdot \mathrm{PAR} \cdot \mathrm{ETR}$-factor

The ETR-factor is supposed to account for the fraction of overall incident PAR that is absorbed within PS II. In most published studies, however, no attempt has been made to determine the ETR-factor, which simply has been assumed to correspond to that of a "model leaf," with $50 \%$ of the PAR being distributed to PS II and $84 \%$ of the PAR being absorbed by photosynthetic pigments in a standard leaf (Björkman and Demmig 1987), so that normally a default ETR-factor of 0.42 is applied.

Without detailed knowledge of the true PS II-specific absorbance, ETR can give a rough estimate only of relative photosynthetic electron transport rate. In the case of dilute algae suspensions, where a minor part of overall incident radiation is absorbed, normally rel.ETR is just treated as an intrinsic parameter of the relative rate of PS II turnover. With this kind of approach, rel.ETR is independent of Chl content, just like Y(II), from which it is derived and, hence, essentially describes the relative frequency of charge-separation at PS II reaction centers. LCs of rel.ETR defined in this way provide useful information, as long as the performance of a particular organism is studied using a fixed color of light, as is the case with standard PAM fluorometers. For this purpose, standard PAM-software provides routines for fitting

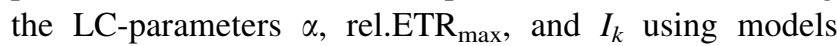
developed by Eilers and Peeters (1988) or Platt et al. (1980). The parameter $\alpha$ relates to the maximal PS II quantum yield (initial slope of LC). Rel.ETR max $_{\text {is a measure of maximal }}$ relative rate and $I_{k}$ relates to the PAR at which light saturation sets in (defined by $\mathrm{ETR}_{\max } / \alpha$ ). For example, diurnal changes in rel.ETR $\mathrm{max}_{\text {(measured with the same sample in its natural }}$ environment) provide valuable information on changes of photosynthetic capacity due to light-dependent enzyme regulation and down-regulation of PS II upon exposure to excess light (Ralph et al. 1999).

While most PAM fluorometers so far have been providing just one color of ML (red or blue) and AL (normally white, red or blue), with the new multi-color-PAM light response curves of the same sample can be recorded using different colors. As expected, in this case substantial differences in LC-parameters are revealed, when a default value of 0.42 is applied as ETR-factor. In Fig. 4, LCs of rel.ETR in Chlorella with 3-min illumination steps using 440- and 625-nm light are compared.

With 440-nm light the rel.ETR LC saturates at much lower PAR than with $625-\mathrm{nm}$ light and the rel.ETR max $_{\text {ax }}$ measured with $440 \mathrm{~nm}$ is much lower than when measured with $625 \mathrm{~nm}$. Furthermore, with $440 \mathrm{~nm}$ after reaching maximal values of rel.ETR, there is some decline of rel.ETR, which is not apparent with $625-\mathrm{nm}$ illumination. The decline of rel.ETR is likely to reflect photoinhibition and, hence, the observed differences between 440- and $625-\mathrm{nm}$ illumination seem to agree with previous findings that blue light is more effective than red light in causing photoinhibition. At this stage, however, it would be premature to interpret these data as evidence for the two-step hypothesis of photoinhibition (see "Introduction"), with the rate-limiting step consisting of blue-light-induced damage of the OEC. Obviously, 440-nm photons are much better absorbed by PS II than $625-\mathrm{nm}$ photons, so that the data also agree with the notion that the extent of photoinhibition increases with the rate of PS II turnover. The decisive question is whether more photoinhibition is also observed when the same flux density of PS II-absorbed 440- and 625-nm photons is applied. This aspect will be further investigated below (see Figs. 8, 9). 


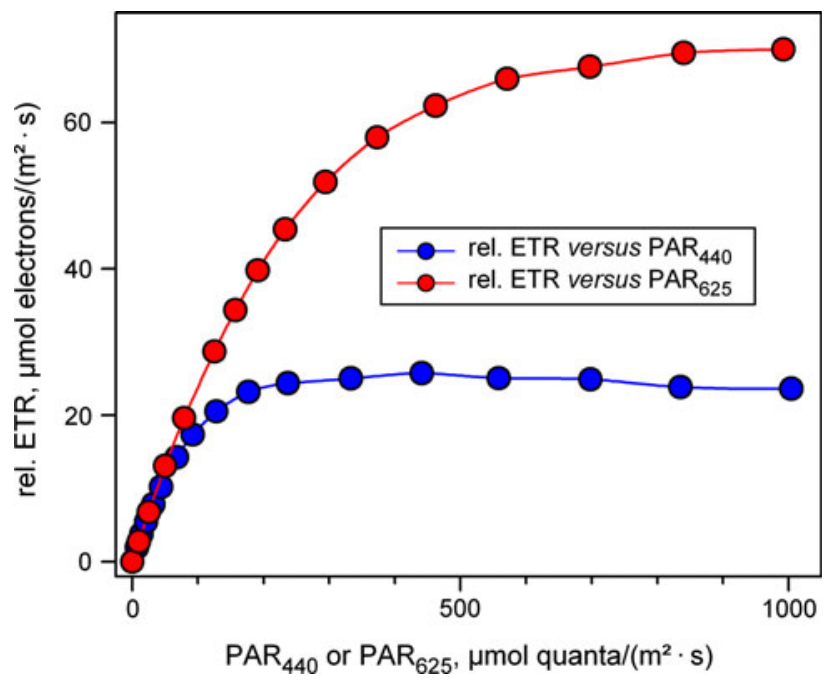

Fig. 4 LC of rel.ETR measured with a dilute suspension of Chlorella (300 $\mu \mathrm{g} \mathrm{Chl/L)} \mathrm{using} \mathrm{440-} \mathrm{and} \mathrm{625-nm} \mathrm{light.} \mathrm{Ignoring} \mathrm{information} \mathrm{on}$ the fraction of incident light absorbed by PS II, a default ETR-factor of 0.42 was applied (see text for explanation and Fig. 8 for comparison). Illumination time at each intensity-setting was $3 \mathrm{~min}$

In Fig. 5, the wavelength-dependent LC-parameters for all five colors are displayed, as derived from consecutive measurements of rel.ETR LC at 440, 480, 540, 590, and $625 \mathrm{~nm}$, with consequent software-assisted fitting of the various LC-parameters according to the model of Eilers and Peeters (1988).

These data show that the same quantum flux density of differently colored light within the range of "PAR" can have vastly different effects, not only between differently pigmented organisms but also within the same organism. Notably, in Chlorella there are even considerable differences between the two types of blue light (440 and $480 \mathrm{~nm})$.

Rel.ETR $\max _{\max }$ and $I_{k}$ display almost identical wavelength dependency, in the case of Chlorella with peak and minimal values at 540 and $440 \mathrm{~nm}$, respectively. The ETR max $_{\text {max }}$ and $I_{k}$ spectra resemble inverse $F_{\mathrm{o}} / \mathrm{PAR}$ spectra (see Fig. 2). It should be kept in mind, however, that PS I contributes to $F_{\mathrm{o}}$, and that rel.ETR $\max _{\text {ax }}$ as well as $I_{k}$ not only depend on PS II but also on PS I activity.

The multi-color-PAM has opened the way for detailed studies of electron transport as a function of the color of light in photosynthetic organisms with largely different pigment compositions. From the above data it is obvious that for such measurements, either a wavelength- and sample-dependent ETR-factor has to be defined or the quantum flux density of PAR has to be replaced by a PS IIspecific quantum flux rate, PAR(II). The latter approach is advantageous, as it results in determination of an absolute rate, independent of chlorophyll content. It requires information on the wavelength- and sample-dependent functional absorption cross section of PS II, Sigma(II) $\lambda$.

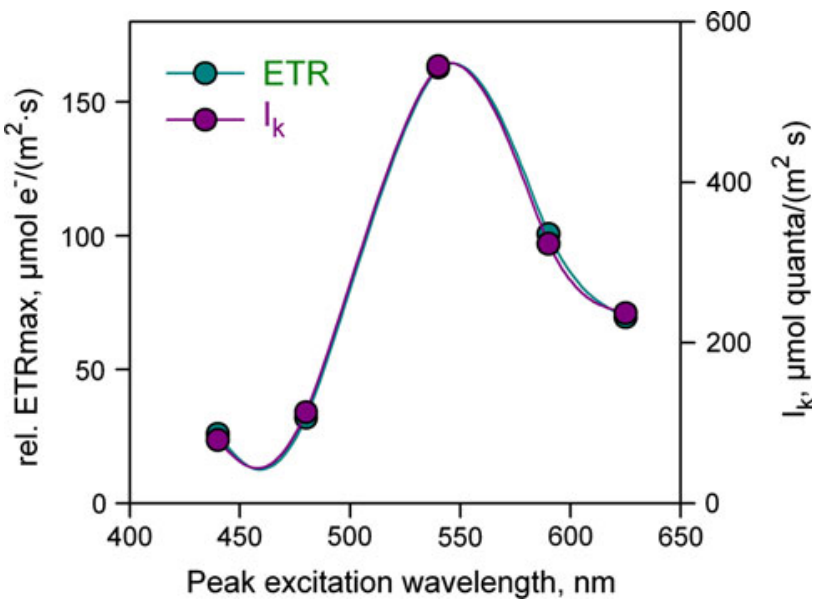

Fig. 5 Rel.ETR $_{\max }$ and $I_{k}$ values of Chlorella plotted against the peak wavelength of the AL. Rel.ETR LCs were measured with same Chlorella sample using different AL colors and a default ETR-factor of 0.42 . Parameters were fitted by a PamWin-3 routine based on the model of Eilers and Peeters (1988)

PAR and the wavelength-dependent functional absorption cross section of PS II, Sigma(II) $\lambda$

Usually, PAR is defined for wavelengths between 400 and $700 \mathrm{~nm}$ (Sakshaug et al. 1997) in units of $\mu \mathrm{mol} /\left(\mathrm{m}^{2} \mathrm{~s}\right)$. It is determined with calibrated quantum sensors, which measure the overall flux density of incident photons, without making any distinction between photons of different colors, as long as their wavelengths fall into the 400-700-nm PAR range.

Hence, the actual extent of PAR-absorption (whether by PS II or PS I or any other colored constituents) by the photosynthetically active sample normally is not taken into account. While this kind of approach has been widely accepted in the study of leaves, which display relatively flat absorbance spectra and absorb most of the incident light, it is not feasible with dilute suspensions of unicellular algae and cyanobacteria, where PS II excitation by light of different wavelengths may vary by an order of magnitude and only a fraction of the incident light is absorbed.

Rappaport et al. (2007) recently pointed out that the "most commonly used unit for light intensity... $\mu \mathrm{mol}$ of photons $\mathrm{s}^{-1} \mathrm{~m}^{-2} \ldots$ has little experimental value since it cannot reliably be translated into a photochemical rate without knowing the absorbance of the sample, which is rarely the case". Further, the authors note that "there is... a real need for a more relevant unit which should be the number of electrons transferred per unit time and per PS II reaction center." Rappaport et al. (2007) determined the rate of PS II turnover via the rate constant of the fluorescence rise induced in the presence of DCMU. As will be outlined below, for quantitative work with the multi-colorPAM, e.g., analysis of light response curves, we prefer to 
translate the quantum flux density (or photon fluence rate) of PAR into a photochemical rate on the basis of information on PS II absorbance of the sample, obtained via measurements of rapid induction kinetics in the absence of DCMU.

Obviously, the PAR information has to be complemented with information on the PS II efficiency of the applied PAR with respect to a given sample. Such information is contained in the wavelength-dependent functional absorption cross section of PS II, the Sigma(II) $\lambda_{\lambda}$, which depends on both the spectral composition of the applied irradiance (i.e., the AL-color) and the PS II absorption properties of the investigated sample. The value of Sigma(II) $)_{\lambda}$ can be derived from the initial rise of fluorescence yield upon onset of saturating light intensity, which directly reflects the rate at which PS II centers are closed. The rate of charge-separation of open PS II centers, $k(\mathrm{II})$, matches the rate with which photons are absorbed by PS II, which may be defined as PAR(II) (see below). In order to account for the overlapping re-opening of PS II centers by secondary electron transport (reoxidation of $\mathrm{Q}_{\mathrm{A}}^{-}$ by $\mathrm{Q}_{\mathrm{B}}$ ), either a PS II inhibitor-like DCMU has to be added, which is not feasible for in vivo studies, or PAR(II) has to be extremely high, so that the reoxidation can be ignored (Koblizek et al. 2001; Kolber et al. 1998; Nedbal et al. 1999), or the rise kinetics have to be corrected for the reoxidation rate.

The last approach is applied with the multi-color-PAM, which is outlined in detail in a separate publication (Klughammer C, Kolbowski J and Schreiber U, in preparation). Here, just one original measurement with a dilute suspension of Chlorella using 440-nm light is presented, which may serve to outline the principle of the approach.

Figure 6 shows the initial part of the increase of fluorescence yield induced by strong AL (in PAM-literature called $O-I_{1}$ rise). The $O-I_{1}$ rise basically corresponds to the $O-J$ phase of the polyphasic OJIP kinetics that have been described in detail by Strasser and co-workers (for reviews see Strasser et al. 2004; Stirbet and Govindjee 2011). There are, however, essential differences in the measuring techniques and definitions of the characteristic fluorescence levels $I_{1}$ and $J$, which argue for different nomenclatures. The multi-color-PAM allows to use the so-called Fast Trigger Files (see "Materials and methods") for routine measurements of fast kinetics. In the example of Fig. 6, the pulse-modulated ML was triggered with $100 \mathrm{kHz}$ pulsefrequency at $100 \mu$ s before onset of $440 \mathrm{~nm} \mathrm{AL}$. At $1 \mathrm{~ms}$ after onset of $\mathrm{AL}$, a saturating 50- $\mu$ s multi-color ST pulse was applied. The ST pulse closes PS II reaction centers transiently, so that the $I_{1}$-level of fluorescence yield can be determined by extrapolation to $1,050 \mu \mathrm{s}$. $I_{1}$ corresponds to the maximal fluorescence yield that can be reached in the presence of an oxidized PQ-pool (for apparent

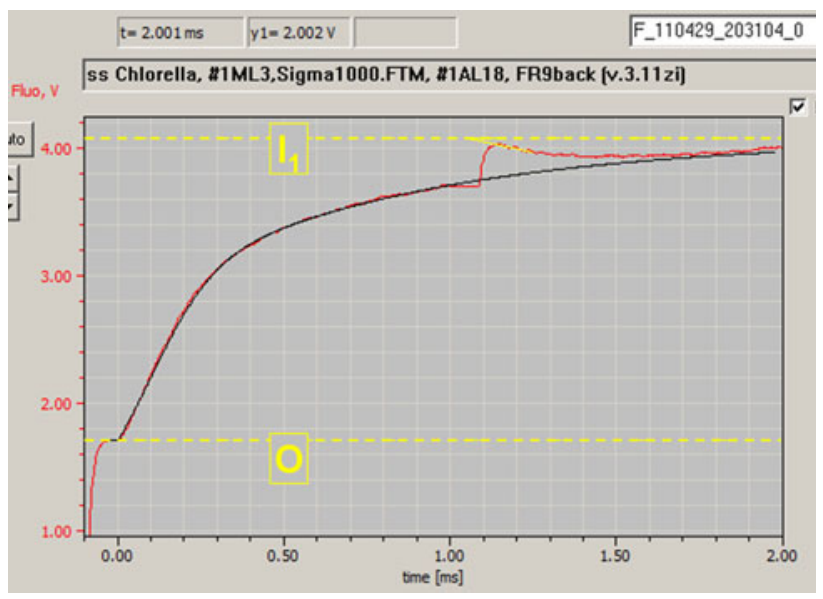

Fig. 6 Initial increase of fluorescence yield $\left(O-I_{1}\right.$ rise) in a dilute suspension of Chlorella (300 $\mu \mathrm{g} \mathrm{Chl/L)}$ induced by $440-\mathrm{nm}$ AL with $2,131 \mu \mathrm{mol}$ quanta/ $\left(\mathrm{m}^{2} \mathrm{~s}\right)$ in presence of FR background light. Dashed yellow lines indicate $F_{\mathrm{o}}$-level $(\mathrm{O})$, assessed during a $50-\mu \mathrm{s}$ period preceding onset of $\mathrm{AL}$ at time zero, and the $I_{1}$-level that is determined with the help of a saturating single-turnover pulse (ST) triggered $1 \mathrm{~ms}$ after onset of AL (see Fig. 2 for the Fast Kinetics trigger pattern). The slope of the relaxation kinetics is extrapolated to the end of the 50- $\mu \mathrm{s} \mathrm{ST}$. The black line represents the $O-I_{1}$ fit curve based on a PS II model which incorporates energy transfer between PS II units and reoxidation of the primary PS II acceptor $\mathrm{Q}_{\mathrm{A}}$ (see text)

PQ-quenching see Samson et al. 1999; Schreiber 2004). Weak FR background light or short FR-preillumination is routinely applied to assure a fully oxidized PQ-pool. This aspect is particularly important in the study of algae and cyanobacteria, where depending on conditions the PQ-pool becomes more or less reduced in the dark via NADPHdehydrogenase activity, resulting in more or less transition into state 2. Furthermore, FR-preillumination minimizes the contribution of "inactive PS II" to the $O-I_{1}$ kinetics.

At a first approximation, assuming that the AL-driven increase of fluorescence yield is linearly correlated with accumulation of $\mathrm{Q}_{\mathrm{A}}^{-}$, and that the initial rise is negligibly slowed down by $\mathrm{Q}_{\mathrm{A}}^{-}$reoxidation, the kinetics can be described by a first order reaction, of which the time constant Tau $=1 / k(\mathrm{II})$ corresponds to the time for reaching a $\mathrm{Q}_{\mathrm{A}}$-reduction level of $100(1-1 / e)=63.2 \%$. When this approximation is applied to the $O-I_{1}$ rise of Fig. 6, Tau $=0.379 \mathrm{~ms}$ is estimated. A thorough analysis of the $O-I_{1}$ rise kinetics, however, has to take into account both $\mathrm{Q}_{\mathrm{A}}^{-}$reoxidation and nonlinearity between $\Delta F$ and the fraction of reduced $Q_{\mathrm{A}}$. This can be achieved by a fitting routine we have specially developed for this purpose (see "Materials and methods").

For the $O-I_{1}$ rise displayed in Fig. 6, which was driven by $2,131 \mu \mathrm{mol}$ quanta/ $\left(\mathrm{m}^{2} \mathrm{~s}\right)$ of $440-\mathrm{nm} \mathrm{AL}$, the following values were estimated by the $O-I_{1}$ fit routine:

Tau $=0.173 \mathrm{~ms}, \quad k(\mathrm{II})=1 / \mathrm{Tau}=5.78 \times 10^{3} / \mathrm{s}, \quad$ Tau $($ reox $)=0.340 \mathrm{~ms}, J=2.01$ (corresponding to $p=0.67$ ), Sigma $(\text { II })_{440}=4.51 \mathrm{~nm}^{2}$. 
Consecutive measurements of $O-I_{1}$ rise kinetics using $440,480,540,590$, and $625 \mathrm{~nm}$ with the same sample are facilitated by the so-called Script-files (see "Materials and methods"). The kinetic parameters of all five rise curves can be fitted together. An example of the obtained data for a dilute suspension of Chlorella is presented in Table 2, which also shows analogous data for Synechocystis.

The fits of Table 2 were carried out under the assumption that the values of the connectivity parameter, $J$, and of the $\mathrm{Q}_{\mathrm{A}}^{-}$reoxidation time constant, Tau(reox) are equal for all colors. It may be noted that the values of the $\mathrm{Q}_{\mathrm{A}^{-}}$ reduction time constant, Tau, were similar for all colors, whereas the applied photon flux rates, PAR, were vastly different. For both the organisms the settings of AL and MT pulse intensities on purpose were programmed to induce rise kinetics with similar initial slopes for all colors. At constant Tau the wavelength-dependent absorption cross section is inversely proportional to the applied PAR (for calculation of Sigma(II), see "Materials and methods"), which is always true, independently of the underlying model of PS II primary reactions. Therefore, with this kind of approach, potential errors due to deficiencies in our model are minimized. Obviously, this approach heavily relies on accurate values of PAR within the sample. For this purpose, the multi-color-PAM features detailed PARlists (see "Materials and methods"), for measurement of which an automated routine is provided.

In Fig. 7, plots of Sigma(II) $)_{\lambda}$ as a function of the peak wavelength are presented for Synechocystis and Chlorella. As expected, these plots resemble fluorescence excitation spectra, similar to the plots of $F_{\mathrm{o}} / \mathrm{PAR}$ presented in Fig. 3A. On closer inspection, comparison of the $F_{\mathrm{o}} / \mathrm{PAR}$ and Sigma(II) $)_{\lambda}$ spectra reveals that there are significant differences for Synechocystis and much less for Chlorella. In Synechocystis, the ratio of maximal to minimal Sig$\mathrm{ma}(\mathrm{II})$ (at 625 and $480 \mathrm{~nm}$, respectively) is 26.1, whereas the corresponding ratio of $F_{\mathrm{o}} / \mathrm{PAR}$ amounts to 15.5 . On the other hand, in Chlorella the ratio of maximal to minimal Sigma(II) (at 440 and $540 \mathrm{~nm}$ ) is 6.0, as compared to 5.1 of the corresponding $F_{\mathrm{o}} / \mathrm{PAR}$. This finding confirms that Sigma(II $)_{\lambda}$ is a more specific measure of PS II excitation than $F_{\mathrm{o}} / \mathrm{PAR}$. While $F_{\mathrm{o}}$ may contain more or less non-PS II fluorescence, depending on excitation wavelength and organism, variable fluorescence yield and the rate with which it is induced, are specific for PS II. Another important difference between Sigma(II) and $F_{\mathrm{o}} / \mathrm{PAR}$ is that Sigma(II) gives absolute information on the functional absorption cross section of PS II, which is independent of $\mathrm{Chl}$ content, whereas $F_{\mathrm{o}} / \mathrm{PAR}$ is proportional to both $\mathrm{Chl}$ content and functional cross section of PS II. Furthermore, $F_{\mathrm{o}} / \mathrm{PAR}$ depends on ML-intensity and gain parameters, which have no influence on Sigma(II), as measured with the multi-color-PAM.

\section{Definition of PAR(II) and ETR(II)}

The wavelength-dependent rate, with which photons (or quanta) are absorbed by PSII, is directly reflected in the $k(\mathrm{II})$ determined by fitting the $O-I_{1}$ rise kinetics measured at high PAR under defined control conditions (see text accompanying Fig. 6). There is direct correspondence between the PS II turnover rate, $k(\mathrm{II})$, in units of electrons/ (PS II s) and the quantum absorption rate at PS II reaction centers in units of quanta/(PS II s). We propose the name PAR(II) for the latter, with the general definition derived from Eq. 1 (see "Materials and methods")

Table 2 Data from consecutive measurements of $O-I_{1}$ rise kinetics in Chlorella vulgaris and Synechocystis PCC 6803

\begin{tabular}{|c|c|c|c|c|c|c|c|}
\hline \multicolumn{8}{|l|}{ Parameter } \\
\hline Peak wavelength (nm) & $F_{\mathrm{o}}(\mathrm{V})$ & $I_{1}(\mathrm{~V})$ & $\operatorname{PAR}\left(\mu \mathrm{mol} /\left(\mathrm{m}^{2} \mathrm{~s}\right)\right)$ & $J$ & Tau (ms) & Tau(reox) (ms) & $\operatorname{Sigma}(\mathrm{II})\left(\mathrm{nm}^{2}\right)$ \\
\hline \multicolumn{8}{|l|}{ Chlorella vulgaris } \\
\hline 440 & 2.199 & 4.981 & 1579 & 2.043 & 0.231 & 0.341 & 4.547 \\
\hline 480 & 2.237 & 5.198 & 2160 & 2.043 & 0.229 & 0.341 & 3.353 \\
\hline 540 & 2.375 & 5.302 & 9649 & 2.043 & 0.228 & 0.341 & 0.756 \\
\hline 590 & 2.293 & 5.205 & 6125 & 2.043 & 0.238 & 0.341 & 1.138 \\
\hline 625 & 2.053 & 4.710 & 4426 & 2.043 & 0.225 & 0.341 & 1.669 \\
\hline \multicolumn{8}{|l|}{ Synechocystis PCC 6803} \\
\hline 440 & 3.193 & 5.243 & 2679 & 2.232 & 0.543 & 0.521 & 1.141 \\
\hline 480 & 3.245 & 4.752 & 9358 & 2.232 & 0.538 & 0.521 & 0.330 \\
\hline 540 & 3.273 & 4.898 & 1907 & 2.232 & 0.537 & 0.521 & 1.621 \\
\hline 590 & 3.232 & 4.943 & 634 & 2.232 & 0.511 & 0.521 & 5.123 \\
\hline 625 & 3.265 & 5.037 & 382 & 2.232 & 0.506 & 0.521 & 8.597 \\
\hline
\end{tabular}

Tau values (time constant of $\mathrm{Q}_{\mathrm{A}}$-reduction) were separately fitted for the five colors, whereas common fits of Tau(reox) (time constant of $\mathrm{Q}_{\mathrm{A}}$ oxidation) and $J$ (connectivity) were applied 


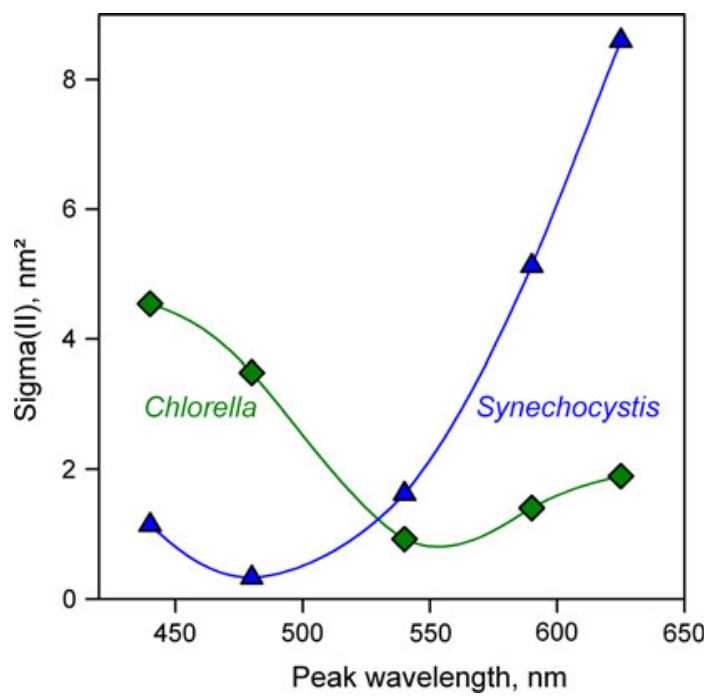

Fig. 7 Functional cross section of PS II, Sigma(II) as a function of AL-color in dilute suspensions $(300 \mu \mathrm{g} \mathrm{Chl} / \mathrm{L})$ of Chlorella and Synechocystis, derived from automated measurements of five consecutive $O-I_{1}$ rise curves each (Script-files Sigma1000Chlor_10.prg and Sigma1000Sycy_10.prg) in the presence of FR background light. Time between consecutive $O-I_{1}$ measurements, $10 \mathrm{~s}$. Sigma(II) values derived by dedicated PamWin-3 fitting routine (see text and Table 2)

$\operatorname{PAR}(\mathrm{II})=k(\mathrm{II})=\operatorname{Sigma}(\mathrm{II})_{\lambda} \cdot L \cdot \mathrm{PAR}$,

where $k(\mathrm{II})$ is the rate constant of PS II turnover, Sigma(II) $)_{\lambda}$ is the functional cross section of PS II (in units of $\mathrm{nm}^{2}$ ), $L$ is Avogadro's constant (with the dimension of $\mathrm{mol}^{-1}$ ), PAR is quantum flux density (or photon fluence rate) and PAR(II) is the rate of quantum absorption in PS II, in units of quanta/(PS II s).

In practice, calculation of PAR(II) from PAR is quite simple when Sigma(II) $)_{\lambda}$ is known: the numerical value of PAR (in units of $\mu \mathrm{mol}$ quanta/ $\left(\mathrm{m}^{2} \mathrm{~s}\right)$ ) just has to be multiplied by $0.6022 \times \operatorname{Sigma}(I I)_{\lambda}$. Hence, once Sigma(II) has been determined for a particular color and sample (via measurement of the $O-I_{1}$ rise kinetics at a defined high light intensity), PAR(II) can be derived for any other PAR (at constant color and state of the sample), without further measurements of fast kinetics. In the case of Chlorella, with Sigma(II) $)_{625}=1.669$ (see Table 2), PAR(II) practically equals PAR, as $0.6022 \times 1.669$ happens to be very close to unity.

An essential difference between PAR(II) and PAR is that the former relates to the quantum absorption rate of PS II, which is independent of Chl content, whereas the latter represents a quantum flux density (or photon fluence rate), from which a PS II quantum absorption rate can be calculated only, if the PS II content is known.

Consequently, based on PAR(II), also a wavelength- and sample-dependent ETR(II) can be defined
$\operatorname{ETR}(\mathrm{II})=\operatorname{PAR}(\mathrm{II}) \cdot \frac{\mathrm{Y}(\mathrm{II})}{\mathrm{Y}(\mathrm{II})_{\max }}$,

where PAR(II) is the rate of quantum absorption at PS II, Y(II) the effective PS II quantum yield derived from the fluorescence ratio parameter $\left(F_{\mathrm{m}}^{\prime}-F\right) / F_{\mathrm{m}}^{\prime}, \mathrm{Y}(\mathrm{II})_{\max }$ the PS II quantum yield in the quasi-dark reference state under which Sigma(II) ${ }_{\lambda}$ was determined and ETR(II) the rate of electron transport expressed in units of electrons/(PS II s).

At very low light intensity, Y(II) approaches $\mathrm{Y}(\mathrm{II})_{\max }$, so that $\mathrm{Y}(\mathrm{II}) / \mathrm{Y}(\mathrm{II})_{\max }=1$ and $\mathrm{ETR}(\mathrm{II})=\mathrm{PAR}(\mathrm{II})$. This means that in this state there is no loss of PS II efficiency with respect to the reference quasi-dark state (all centers open, non-energized, weak FR background illumination) under which $\operatorname{Sigma}(\mathrm{II})_{\lambda}$ was measured. Y(II) $)_{\max }$ corresponds to the PS II quantum yield of a sample in the same state as given for measurement of $k(\mathrm{II})$, which equals $F_{\mathrm{v}} /$ $F_{\mathrm{m}}$. In measurements with algae and cyanobacteria, which display a relatively high level of PQ-reduction in the dark, it is advisable to measure $F_{\mathrm{v}} / F_{\mathrm{m}}$ in the presence of FR background light, which oxidizes the PQ-pool and induces the high PS II-efficiency state 1. FR background light is also routinely used for assessment of $k(\text { II) and Sigma(II) })_{\lambda}$ via the $O-I_{1}$ rise kinetics.

2 When compared with the common definition of rel.ETR in Eq. 2, it is apparent that the ETR-factor is contained in PAR(II) and that ETR(II) has the dimension of a turnover rate per PS II, whereas rel.ETR commonly has been treated as an electron flux density (or fluence rate), i.e., a rate per area, which without information on PS II per area must be considered hypothetical. In contrast, ETR(II) realistically describes the mean absolute rate of charge-separation per PS II in all PS II contained in the 1-mL illuminated sample.

When the appropriate wavelength- and sample-dependent Sigma(II) ${ }_{\lambda}$ value is known, the user software of the multi-color-PAM supports the transformation of PAR into PAR(II). A practical example of transformation of a PARscale into a PAR(II) scale is given in Fig. 8, which is derived from the original rel.ETR LC data of Fig. 4 using the information on the values of $\operatorname{Sigma(II)}{ }_{\lambda}$ measured with the same dilute Chlorella suspension briefly before the LC recording. PAR values were transformed into PAR(II) using Eq. 3 and ETR(II) was calculated according to Eq. 4.

In contrast to the rel.ETR LC of Fig. 4, where rel.ETR ${ }_{\max }$ was much higher for $625 \mathrm{~nm}$ than for $440 \mathrm{~nm}$, the ETR(II) max $_{\max }$ values in Fig. 8 are almost identical for both the colors, thus confirming that the observed differences in rel.ETR are almost exclusively due to differences between Sigma(II) 440 and Sigma(II) ${ }_{625}$. This may be considered strong support for the validity of Sigma(II) $\lambda_{\lambda}$ determination via $O-I_{1}$ measurements with the multi-colorPAM and its analysis by the $O-I_{1}$ Fit approach. As the maximal value of ETR(II) $)_{440}$ is slightly lower than that of 


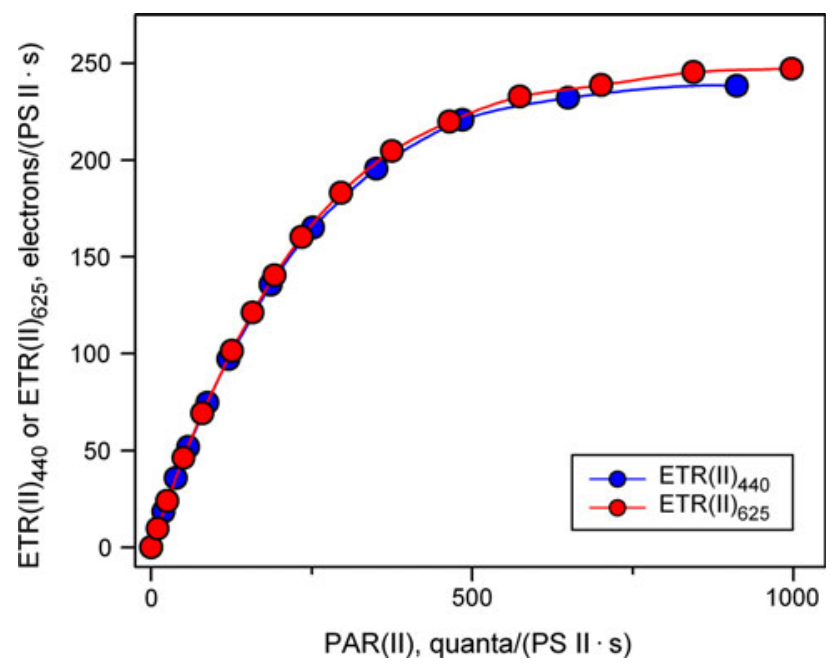

Fig. 8 ETR(II) LC of a dilute suspension of Chlorella $(300 \mu \mathrm{g} \mathrm{Chl} /$ L) using 440- and 625-nm light derived from the original LC of rel.ETR depicted in Fig. 4 by transformation of the PAR-scale into a PAR(II) scale using Eqs. 3 and 4 (see text). Illumination time at each intensity-setting was $3 \mathrm{~min}$. Sigma(II) values of 4.547 and $1.669 \mathrm{~nm}^{2}$ were applied for 440 and $625 \mathrm{~nm}$, respectively. In the calculation of ETR(II) ${ }_{440}$ and ETR(II) $)_{625}, F_{\mathrm{v}} / F_{\mathrm{m}}$ values of 0.68 and 0.66 were used, respectively. For comparison of the corresponding LC without PAR transformation, see Fig. 4

ETR(II) ${ }_{625}$, the question remains whether even after transformation of PAR into PAR(II), i.e., for identical rates of PS II turnover, blue light causes somewhat more photoinhibition (or down-regulation) than red light. For evaluation of these results it has to be considered that the illumination periods during the LC recording were relatively short $(3 \mathrm{~min})$, so that the time of exposure to potentially photoinhibitory intensities was relatively short. This aspect is further investigated in the following section.

When information on PS II concentration is available, it is possible to derive from ETR(II) a rough estimate of the absolute $\mathrm{O}_{2}$ evolution rate in units of $\mathrm{mmol} \mathrm{O}_{2} /(\mathrm{mg} \mathrm{Chl} \mathrm{s})$ using the following general equation:

$r \mathrm{O}_{2}=\frac{\mathrm{ETR}(\mathrm{II})}{\mathrm{PSU} \cdot n e\left(\mathrm{O}_{2}\right) \cdot M(\mathrm{Chl})}$,

where PSU is the photosynthetic unit size (i.e., number of Chl molecules per electron transport chain), $M(\mathrm{Chl})$ is the molecular weight of Chl (approximately $900 \mathrm{~g} / \mathrm{mol}$ ) and $n e\left(\mathrm{O}_{2}\right)$ the number of electrons required for evolution of 1 molecule of $\mathrm{O}_{2}$ (normally assumed to be 4). The absolute rate in the common units of $\mu \mathrm{mol} \mathrm{O}_{2} /(\mathrm{mg} \mathrm{Chl} \mathrm{h})$ is obtained by multiplication with $1,000 \times 3,600$. If $\mathrm{PSU}=1,000$ is assumed, the numerical value of the denominator amounts to $1,000 \times 3,600$, which means that in this case the numerical values of ETR(II) in electrons/ (PS II s) and $r \mathrm{O}_{2}$ in $\mu \mathrm{mol} \mathrm{O}_{2} /(\mathrm{mg} \mathrm{Chl} \mathrm{h})$ are identical.
Comparison of photoinhibition by 440- and $625-\mathrm{nm}$ illumination

The Chlorella cells used in this study were cultured at relatively low ambient light intensities in the order of 20-30 $\mu$ mol quanta/ $\left(\mathrm{m}^{2} \mathrm{~s}\right)$ PAR, which may be compared with the $I_{k}$ values of Chlorella, i.e., with the PAR values were light saturation sets in (see Fig. 5) that were 80 and $214 \mu \mathrm{mol} /\left(\mathrm{m}^{2} \mathrm{~s}\right)$ for 440 and $625 \mathrm{~nm}$, respectively. The maximal intensities applied in the experiment of Figs. 4, 5, and 8 amounted to $1,000 \mu \mathrm{mol} /\left(\mathrm{m}^{2} \mathrm{~s}\right)$ for both the colors. Hence, in view of the up to about 50 times higher light intensities during LC recordings compared to growth conditions, photoinhibitory damage would not be surprising. In discussing Fig. 8, the question was raised, whether the slightly lower ETR(II) $)_{\max }$ values with $440 \mathrm{~nm}$ compared to $625 \mathrm{~nm}$ could be due to a somewhat stronger photoinhibitory effect of $440 \mathrm{~nm}$, as predicted by the twostep hypothesis of photoinhibition (see "Introduction"). This question can be further investigated by comparative measurements of dark-light-dark induction curves with repetitive assessment of effective PS II quantum yield, Y(II), where Chlorella is exposed for a longer period of time $(22 \mathrm{~min})$ to relatively high intensities of 440 - and 625-nm light.

The data in Fig. 9 were obtained by automated measurements of slow kinetics under the control of a "Scriptfile" (see "Materials and methods") programmed for initial measurement of $F_{\mathrm{v}} / F_{\mathrm{m}}=\mathrm{Y}(\mathrm{II})_{\max }$ and 22 min continuous illumination followed by 50-min dark-regeneration, with SPs applied every 5 min for determination of effective PS II quantum yield, Y(II). The 22-min continuous illumination served as photoinhibitory treatment and during the 50 min following this treatment the multi-phasic recovery of Y(II) was monitored. The Script was run four times with fresh samples using three different intensities of $440 \mathrm{~nm}$ and a single intensity of $625-\mathrm{nm}$ light. The PAR of the 625-nm light was chosen such that it induced close to the same rate of PS II turnover as the medium intensity of the 440 -nm light, i.e., the same PAR(II) was applied, as derived by Eq. 3 (in the given example, $419 \times 4.547$ almost equals $1,088 \times 1.669)$.

Comparison of the three curves with 440-nm illumination (dark-blue curve at top and two light-blue curves at bottom of Fig. 9) provides some insight into light-induced suppression of $\mathrm{Y}$ (II) in Chlorella. At $80-\mu \mathrm{mol} /\left(\mathrm{m}^{2} \mathrm{~s}\right)$ (top curve, corresponding to $I_{k}$, i.e., near the beginning of saturation) after its initial suppression Y(II) gradually increases during illumination, reflecting light-activation of the Calvin-Benson cycle. Upon darkening, Y(II) returns with biphasic kinetics within $50 \mathrm{~min}$ to its original darklevel. In contrast, at $419 \mu \mathrm{mol} /\left(\mathrm{m}^{2} \mathrm{~s}\right)$ (third curve from top) not only the initial suppression of Y(II) is more pronounced 


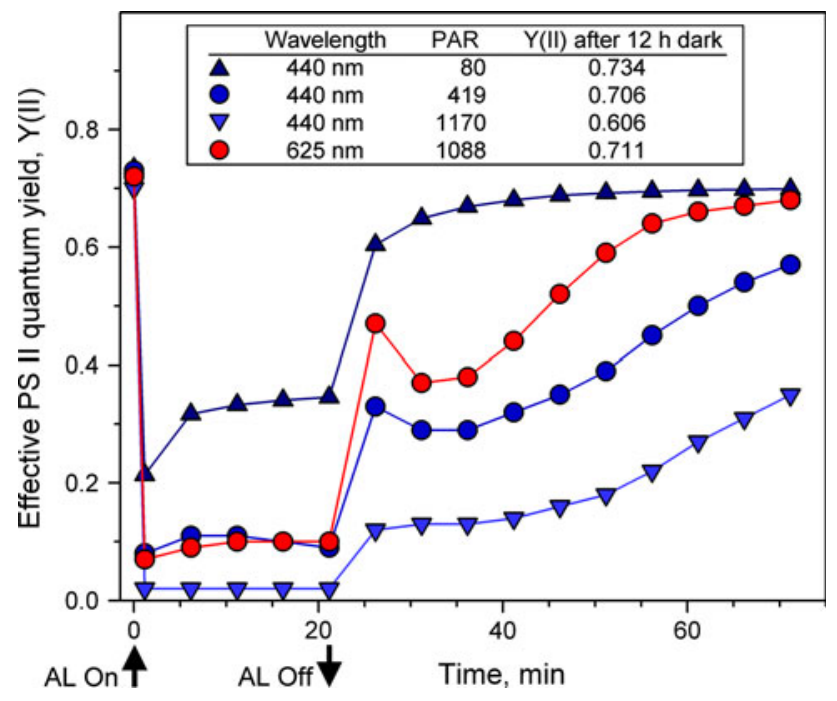

Fig. 9 Changes of effective quantum yield, Y(II), induced during 22-min illumination with 440- and 625-nm light in dilute suspensions of Chlorella (300 $\mu \mathrm{g} \mathrm{Chl/L})$ followed by 50 -min dark-regeneration. $\mathrm{AL}$ was switched on $40 \mathrm{~s}$ after measurement of $F_{\mathrm{v}} / F_{\mathrm{m}}$ (at time 0 ) and SP were applied every $5 \mathrm{~min}$, starting $20 \mathrm{~s}$ after onset of AL. Use of the Script-file photoinhibition_Chl01.prg, with settings of light color and AL-intensity varied. PAR values are indicated in $\mu$ mol quanta/ $\left(\mathrm{m}^{2} \mathrm{~s}\right)$

but also after about $10 \mathrm{~min}$ there is a gradual decline of Y(II), which suggests that light-activation of the CalvinBenson cycle cannot prevent gradually increasing inhibition of PS II. Upon darkening, a rapid phase of Y(II) recovery is followed by a dip phase, before Y(II) slowly returns toward its dark-level, with distinctly slower kinetics than in the experiment using $80 \mu \mathrm{mol} /\left(\mathrm{m}^{2} \mathrm{~s}\right)$ and $80 \%$ dark-recovery within $50 \mathrm{~min}$. Finally, at $1,022 \mu \mathrm{mol} /\left(\mathrm{m}^{2} \mathrm{~s}\right)$ of 440-nm light (bottom curve), Y(II) is suppressed to values close to zero during illumination and the recovery upon darkening displays two phases separated by a wide plateau, with 50-min dark-recovery amounting to $49 \%$ only. After 12-h dark-recovery, the Y(II) of all samples except for the one illuminated at $1,170 \mu \mathrm{mol} /\left(\mathrm{m}^{2} \mathrm{~s}\right)$ recovered to values close to the original $F_{\mathrm{v}} / F_{\mathrm{m}}$ (see inset of Fig. 9).

The red curve in Fig. 9 shows the responses observed when almost identical PAR(II) of 625-nm light is applied $\left(1,088 \mu \mathrm{mol} /\left(\mathrm{m}^{2} \mathrm{~s}\right)\right)$ as in the measurement with the intermediate $440-\mathrm{nm}$ intensity $\left(419 \mu \mathrm{mol} /\left(\mathrm{m}^{2} \mathrm{~s}\right)\right)$. Hence, at equal PAR(II), the responses of 440- and 625-nm quanta are very similar, even when applied over a longer period of time. At the end of illumination the Y(II) with $625 \mathrm{~nm}$ is just marginally higher than with $440 \mathrm{~nm}$, similarly as in the LC-recordings of Fig. 8. There are, however, remarkable differences in the dark-recovery kinetics. After 625-nm illumination, the dark-recovery of $\mathrm{Y}(\mathrm{II})$ is distinctly faster than after 440-nm illumination, amounting to $97 \%$ after $50 \mathrm{~min}$. This shows clearly that the PS II turnover is not the only parameter affecting the quantum yield of PS II. Obviously, $440 \mathrm{~nm}$ can lower the PS II quantum yield by an additional mechanism, which is induced at high light intensity and still is effective after 50-min dark-recovery.

\section{Concluding discussion and outlook}

The presented data demonstrate that the new multi-colorPAM is more than just another PAM fluorometer offering various colors of light. The new dimension of this device relates to the precision, flexibility, and speed, with which the various colors of light can be applied, with the main aim of obtaining quantitative information on the rate of wavelength-dependent charge-separation in PS II reaction centers. This aim was reached via new developments at the levels of opto-electronics, microprocessor-based firmware and user software. Recent technical progress in LED technology made it possible to develop an extremely powerful miniature light source, which provides all the essential light qualities, for which in former days a whole bench of high-power light sources and flash-discharge lamps (or lasers) would have been required. With six separate colors of ML, six colors of AL (also serving for ST and MT flashes) and FR light, a total of 13 independent light sources are integrated on the $10 \times 10 \mathrm{~mm}$ area of the multi-color-COB array. Such compact design enables optimal coupling of the light source to the $10 \times 10 \mathrm{~mm}$ sample cuvette, assuring identical optical pathways of the various types of light. Close to optimal optical conditions are possible by use of low cell densities, as excellent signal/noise ratios are obtained at $200-300 \mu \mathrm{g} \mathrm{Chl} / \mathrm{L}$, where light-intensity gradients are negligibly small. Operation of this complex measuring system is facilitated by automated measuring routines, which assure reproducibility and prevent operator's errors.

The novel information provided by the new device is contained in the wavelength-dependent parameter Sig$\operatorname{ma}(\mathrm{II})_{\lambda}$, the definition of which for technical-methodological reasons differs from the parameter $\sigma_{\mathrm{PSII}}$ used by researchers in limnology and oceanography (Koblizek et al. 2001; Kolber et al. 1998). Almost all $\sigma_{\text {PSII }}$ values reported in the literature were determined for one color of light, irrespective of the pigment-composition of the investigated sample. Furthermore, $\sigma_{\mathrm{PSII}}$ has been measured in widely differing states of the sample, with the PS II acceptor side being more or less reduced, which leads to corresponding changes in the sigmoidicity and time constant of the lightinduced fluorescence rise. In contrast, Sigma(II) ${ }_{\lambda}$ is always measured in a defined quasi-dark reference state, at close to maximal efficiency of PS II. Any changes of the sample with respect to this reference state, e.g., by light-driven down-regulation or photodamage of PS II, do not affect 
Sigma(II) ${ }_{\lambda}$, but are contained in the effective PS II quantum yield, Y(II), which is lowered with respect to the PS II quantum yield, $\mathrm{Y}(\mathrm{II})_{\max }$, measured in the reference state, in which also Sigma(II) $)_{\lambda}$ was measured. Therefore, the values of Sigma(II $)_{\lambda}$ obtained for Chlorella and Synechocystis are substantially higher than the $\sigma_{\text {PSII }}$ values reported, e.g., by Koblizek et al. (2001).

Other new parameters introduced for work with the multi-color-PAM are PAR(II) and ETR(II), which describe the absolute rates of photon absorption by PS II and electron transport via PS II, respectively. PAR(II) just like $\operatorname{Sigma}(\mathrm{II})_{\lambda}$ is defined for a quasi-dark reference state. With this approach, fluorescence-based estimation of absolute photosynthetic electron transport rates in optically thin suspensions has been given a reliable methodological basis. Related work using the parameter $\sigma_{\text {PSII }}$ can be found almost exclusively in the limnology and oceanography literature, which partially may be due to the complexity of its definition, understanding of which requires considerable background knowledge. Comparison of Figs. 4 and 8 demonstrates convincingly that quantitative information on the functional PS II absorption cross section is of general importance for quantitative assessment of photosynthetic activity, which becomes very evident as soon as different colors of light are applied.

It may be foreseen that the multi-color-PAM will stimulate future research of the wavelength dependence of photosynthesis not only in suspensions of algae and cyanobacteria but also in whole leaves, macrophytes or even corals and other organisms containing endosymbionts. The example presented of apparent differences in photoinhibition by close to identical PAR(II) of 440- and 625-nm light in Chlorella demonstrates the methodological value of Sigma(II $)_{\lambda}$ determination with this new device in a range of basic and applied plant physiological applications. Adjustment of close to equal PAR(II) should be also possible with leaves and other optically dense samples. When fluorescence is excited by 440-nm ML and $F<710 \mathrm{~nm}$ is measured, almost selectively fluorescence responses of the uppermost cell layers are measured (Schreiber et al. 2011), so that differences due to varying depths of penetration can be avoided. This is an example for the advantage of optional use of separate colors for measuring and actinic light. Rappaport et al. (2007) pointed out the advantages of using green light (both measuring and actinic) to minimize light-intensity gradients. However, even with green light substantial gradients persist and, most importantly, the photosynthetic performance of different cell layers within a leaf (as well as other types of optically dense samples) is heterogeneous and their responses should not be mixed up. Therefore, to assess, e.g., differences between adaxial and abaxial leaf sides it is better to employ strongly absorbed ML (e.g., $440 \mathrm{~nm}$ ), so that the response is restricted to the uppermost layers of cells, which may be considered close to homogenous (Schreiber et al. 2011).

The data of Fig. 9 were presented as one example of practical application of the new multi-color device to induce defined rates of quanta absorption in PS II using different colors. These measurements may be considered particularly reliable, as they were carried out with dilute suspensions, i.e., with negligibly small PAR-gradients. The data demonstrate distinct differences between post-illumination responses after close to identical absorption of 440and 625-nm quanta, the direction of which in principle does agree with the two-step hypothesis of photoinhibition. Specific absorption of blue light could cause damage of the Mn-cluster of the OEC, resulting in donor-side limitation of PS II, production of ROS and secondary damage of various enzymatic reactions, including repair of PS II reaction centers (Ohnishi et al. 2005; Hakala et al. 2005; Nishiyama et al. 2006). However, this may not be the only mechanism that can explain the observed differences between 440- and 625-nm light. More extensive measurements, using longer illumination times and inhibition of the simultaneously occurring repair reactions, will be required for conclusive evidence. In any case, it is clear that the multi-color-PAM does offer the potential for quantitative investigation of the wavelength dependence of photoinhibition, particularly when combined with other promising new measuring techniques (Chow et al. 2005; Matsubara and Chow 2004).

Besides the mechanism of photodamage to PS II, other important topics relating to wavelength-dependent effects on the photosynthetic apparatus are reversible state 1-state 2 transitions (Mullineaux and Emlyn-Jones 2005) and NPQ induced in cyanobacteria via blue-light absorption by the orange carotenoid protein (Kirilovsky 2007). The multicolor-PAM appears well suited for detailed investigations of these fascinating adaptational processes (Bernát et al. 2012).

Acknowledgments We thank Erhard Pfündel for fruitful discussions and professional help with the preparation of the manuscript.

Open Access This article is distributed under the terms of the Creative Commons Attribution License which permits any use, distribution, and reproduction in any medium, provided the original author(s) and the source are credited.

\section{References}

Allen MM (1968) Simple conditions for growth of unicellular bluegreen algae on plates. J Phycol 4:1-8

Bernát G, Schreiber U, Sendtko E, Stadnichuk IN, Rexroth S, Rögner M, Koenig F (2012) Unique properties vs. common themes: the atypical cyanobacterium Gloeobacter violaceus PCC 7421 is capable of state transitions and blue-light induced fluorescence quenching. Plant Cell Physiol. doi:10.1093/pcp/pcs009 
Beutler M, Wiltshire KH, Meyer B, Moldaenke C, Lüring C, Meyerhöfer M, Hansen U-P, Dau H (2002) A fluorometric method for the differentiation of algal populations in vivo and in situ. Photosynth Res 72:39-53

Björkman O, Demmig B (1987) Photon yield of $\mathrm{O}_{2}$-evolution and chloroplast fluorescence characteristics at $77 \mathrm{~K}$ among vascular plants of diverse origins. Planta 170:489-504

Braslavsky SE (2007) Glossary of terms used in photochemistry, 3rd edition. Pure Appl Chem 79:293-465

Chow WS, Lee HY, He J, Hendrickson L, Hong YN, Matsubara S (2005) Photoinactivation of photosystem II in leaves. Photosynth Res 84:35-41

Demmig-Adams B, Adams WW III (1992) Photoprotection and other responses of plants to high-light stress. Ann Rev Plant Physiol Plant Mol Biol 43:599-626

Eilers PHC, Peeters JCH (1988) A model for the relationship between light intensity and the rate of photosynthesis in phytoplankton. Ecol Model 42:199-215

Falkowski PG, Kolber Z (1995) Variations in chlorophyll fluorescence yields in phytoplankton in the world oceans. Aust J Plant Physiol 22:341-355

Falkowski PG, Raven JA (2007) Aquatic photosynthesis, 2nd edn. Princeton University Press, Princeton

Falkowski PG, Koblizek M, Gorbunov M, Kolber Z (2004) Development and application of variable fluorescence techniques in marine ecosystems. In: Papageorgiou G, Govindjee (eds) Chlorophyll fluorescence: a signature of photosynthesis. Kluwer, Dordrecht, pp 279-319

Genty B, Briantais J-M, Baker NR (1989) The relationship between the quantum yield of photosynthetic electron transport and quenching of chlorophyll fluorescence. Biochim Biophys Acta 990:87-92

Gilbert M, Domin A, Becker A, Wilhelm C (2000a) Estimation of primary productivity by chlorophyll a in vivo fluorescence in freshwater phytoplankton. Photosynthetica 38:111-126

Gilbert M, Wilhelm C, Richter M (2000b) Bio-optical modelling of oxygen evolution using in vivo fluorescence: comparison of measured and calculated photosynthesis/irradiance $(\mathrm{P}-\mathrm{I})$ curves in four representative phytoplankton species. J Plant Physiol 157:307-314

Hakala M, Tuominen I, Keränen M, Tyystjärvi T, Tyystjärvi E (2005) Evidence for the role of the oxygen-evolving manganese complex in photoinhibition of Photosystem II. Biochim Biophys Acta 1706:68-80

Herlory O, Richard P, Blanchard GF (2007) Methodology of light response curves: application of chlorophyll fluorescence to microphytobenthic biofilms. Marine Biol 153:91-101

Jakob T, Schreiber U, Kirschesch V, Langner U, Wilhelm C (2005) Estimation of chlorophyll content and daily primary production of the major algal groups by means of multiwavelengthexcitation PAM chlorophyll fluorometry: performance and methodological limits. Photosynth Res 83:343-361

Kirilovsky D (2007) Photoprotection in cyanobacteria: the orange carotenoid protein (OCP)-related non-photochemical-quenching mechanism. Photosynth Res 93:7-16

Klughammer C, Schreiber U (2008) Complementary PS II quantum yields calculated from simple fluorescence parameters measured by PAM fluorometry and the Saturation Pulse method. PAM Application Notes, vol 1, pp 27-35. http://www.walz.com/down loads/pan/PAN11001.pdf

Koblizek M, Kaftan D, Nedbal L (2001) On the relationship between the non-photochemical quenching of the chlorophyll fluorescence and the Photosystem II light harvesting efficiency. A repetitive flash fluorescence induction study. Photosynth Res 68:141-152

Kolber ZS, Prášil O, Falkowski PG (1998) Measurement of variable chlorophyll fluorescence using fast repetition rate techniques: defining methodology and experimental protocols. Biochim Biophys Acta 1367:88-106

Kolbowski J, Schreiber U (1995) Computer-controlled phytoplankton analyzer based on 4-wavelengths PAM chlorophyll fluorometer. In: Mathis P (ed) Photosynthesis: from light to biosphere, vol V. Kluwer, Dordrecht, pp 825-828

Krall JP, Edwards GE (1990) Quantum yields of Photosystem II electron transport and carbon dioxide fixation in $\mathrm{C} 4$ plants. Aust J Plant Physiol 17:579-588

Kramer DM, Johnson G, Kiirats O, Edwards GE (2004) New fluorescence parameters for the determination of $\mathrm{Q}_{\mathrm{A}}$ redox state and excitation energy fluxes. Photosynth Res 79:209-218

Lavergne J, Leci E (1993) Properties of inactive PS II centers. Photosynth Res 38:323-343

Lavergne J, Trissl HW (1995) Theory of fluorescence induction in photosystem II: derivation of analytical expressions in a model including exciton-radical-pair equilibrium and restricted energy transfer between photosynthetic units. Biophys J 68:2474-2492

Ley AC, Mauzerall DC (1982) Absolute absorption cross-sections for Photosystem II and the minimum quantum requirement for photosynthesis in Chlorella vulgaris. Biochim Biophys Acta 680:95-106

Matsubara S, Chow WS (2004) Populations of photoinactivated Photosystem II characterized by chlorophyll fluorescence lifetime in vivo. Proc Natl Acad Sci USA 101:18234-18239

Mullineaux CW, Emlyn-Jones D (2005) State transitions: an example of acclimation to low-light stress. J Exp Bot 56:389-393

Nedbal L, Trtílek M, Kaftan D (1999) Flash fluorescence induction: a novel method to study regulation of Photosystem II. J Photochem Photobiol B 48:154-157

Neubauer C, Schreiber U (1987) The polyphasic rise of chlorophyll fluorescence upon onset of strong continuous illumination: I. Saturation characteristics and partial control by the photosystem II acceptor side. Z Naturforsch 42c:1246-1254

Nishiyama Y, Allakhverdiev SI, Murata N (2006) A new paradigm for the action of reactive oxygen species in the photoinhibition of photosystem II. Biochim Biophys Acta 1757:742-749

Oguchi R, Douwstra P, Fujita T, Chow WS, Terashima I (2011) Intraleaf gradients of photoinhibition induced by different color lights: implications for the dual mechanisms of photoinhibition and for the application of conventional chlorophyll fluorometers. New Phytol 191:146-159

Ohnishi N, Allakhverdiev SI, Takahashi S, Higashi S, Watanabe M, Nishiyama Y, Murata N (2005) Two-step mechanism of photodamage to photosystem II: step 1 occurs at the oxygenevolving complex and step 2 occurs at the photochemical reaction center. Biochemistry 44:8494-8499

Osmond CB (1981) Photorespiration and photoinhibition. Some implications for the energetics of photosynthesis. Biochim Biophys Acta 639:77-98

Osmond CB (1994) What is photoinhibition? Some insights from comparisons of shade and sun plants. In: Baker N, Bowyer JR (eds) Photoinhibition of photosynthesis. BIOS Scientific Publishers, Oxford, pp 1-24

Papageorgiou GC, Govindjee (eds) (2004) Chlorophyll fluorescence: a signature of photosynthesis. Springer, Dordrecht

Pirson A, Ruppel HG (1962) Über die induktion einer teilungshemmung in synchronen Kulturen von Chlorella. Arch Mikrobiol 42:499-505

Platt T, Gallegos CL, Harrison WG (1980) Photoinhibition of photosynthesis in natural assemblages of marine phytoplankton. J Marine Res 38:687-701

Ralph PJ, Gademann R (2005) Rapid light curves: a powerful tool to assess photosynthetic activity. Aquat Bot 82:222-237

Ralph PJ, Gademann R, Larkum AWD, Schreiber U (1999) In situ underwater measurements of photosynthetic activity of coral 
zooxanthellae and other reef-dwelling dinoflagellate endosymbionts. Mar Ecol Prog Ser 180:139-147

Rappaport F, Béal D, Joliot A, Joliot P (2007) On the advantage of using green light to study fluorescence yield changes in leaves. Biochim Biophys Acta 1767:56-67

Rascher U, Liebig Lüttge (2000) Evaluation of instant light-response curves of chlorophyll fluorescence parameters obtained with a portable chlorophyll fluorometer on site in the field. Plant Cell Environ 23:1397-1405

Sakshaug E, Bricaud A, Dandonneau Y, Falkowski PG, Kiefer DA, Legendre L, Morel A, Parslow J, Takahashi M (1997) Parameters of photosynthesis: definitions, theory and interpretation of results. J Plankton Res 19:1637-1670

Samson G, Prášil O, Yaakoubd B (1999) Photochemical and thermal phases of chlorophyll a fluorescence. Photosynthetica 37(2): $163-182$

Schreiber U (1986) Detection of rapid induction kinetics with a new type of high-frequency modulated chlorophyll fluorometer. Photosynth Res 9:261-272

Schreiber U (2004) Pulse-amplitude (PAM) fluorometry and saturation pulse method. In: Papageorgiou G, Govindjee (eds) Chlorophyll fluorescence: a signature of Photosynthesis. Kluwer, Dordrecht, pp 279-319

Schreiber U, Krieger A (1996) Hypothesis: two fundamentally different types of variable chlorophyll fluorescence in vivo. FEBS Lett 397:131-135

Schreiber U, Bilger W, Schliwa U (1986) Continuous recording of photochemical and non-photochemical chlorophyll fluorescence quenching with a new type of modulation fluorometer. Photosynth Res 10:51-62

Schreiber U, Neubauer C, Schliwa U (1993) PAM fluorometer based on medium-frequency pulsed Xe-flash measuring light: a highly sensitive new tool in basic and applied photosynthesis research. Photosynth Res 36:65-72

Schreiber U, Bilger W, Neubauer C (1994) Chlorophyll fluorescence as a non-intrusive indicator for rapid assessment of in vivo photosynthesis. In: Schulze E-D, Caldwell MM (eds) Ecological studies, vol 100. Springer, Heidelberg, pp 49-70

Schreiber U, Hormann H, Neubauer C, Klughammer C (1995) Assessment of photosystem II photochemical quantum yield by chlorophyll fluorescence quenching analysis. Aust J Plant Physiol 22:209-220

Schreiber U, Kühl M, Klimant I, Reising H (1996) Measurement of chlorophyll fluorescence within leaves using a modified PAM fluorometer with a fiber-optic microprobe. Photosynth Res 47: 103-109

Schreiber U, Gademann R, Ralph PJ, Larkum AWD (1997) Assessment of photosynthetic performance of prochloron in LissoclinumPatella in hospite by chlorophyll fluorescence measurements. Plant Cell Physiol 38:945-951

Schreiber U, Klughammer C, Kolbowski J (2011) High-end chlorophyll fluorescence analysis with the MULTI-COLOR-PAM. I. Various light qualities and their applications. PAM Application Notes, vol 1, pp 1-19. http://www.walz.com/downloads/pan/PAN11001.pdf

Siebke K, von Caemmerer S, Badger M, Furbank RT (1997) Expressing an $\mathrm{RbcS}$ antisense gene in transgenic Flaveria bidentis leads to an increased quantum requirement for $\mathrm{CO}_{2}$ fixed in Photosystems I and II. Plant Physiol 115:1163-1174

Stirbet A, Govindjee (2011) On the relation between the Kautsky effect (chlorophyll a fluorescence induction) and Photosystem II: basics and applications of the OJIP fluorescence transient. J Photochem Photobiol B 104:236-257

Strasser RJ, Tsimilli-Michael M, Srivastava A (2004) Analysis of the chlorophyll $a$ fluorescence transient. In: Papageorgiou G, Govindjee (eds) Chlorophyll fluorescence: a signature of photosynthesis. Kluwer, Dordrecht, pp 321-362

Suggett DJ, Moore CM, Hickman AE, Geider RJ (2009) Interpretation of fast repetition rate (FRR) fluorescence: signatures of phytoplankton community structure versus physiological state. Mar Ecol Prog Ser 376:1-19

Takahashi S, Milward SE, Yamori W, Evans JR, Hillier W, Badger MR (2010) The solar action spectrum of photosystem II damage. Plant Physiol 153:988-993

Terashima I, Fujita T, Inoue T, Chow WS, Oguchi R (2009) Green light drives leaf photosynthesis more efficiently than red light in strong white light: revisiting the enigmatic question of why leaves are green. Plant Cell Physiol 50:684-697

Trampe E, Kolbowski J, Schreiber U, Kühl M (2011) Rapid assessment of different oxygenic phototrophs and single-cell photosynthesis with multicolour variable chlorophyll fluorescence imaging. Mar Biol 158:1667-1675

Van Kooten O, Snel JFH (1990) The use of chlorophyll fluorescence nomenclature in plant stress physiology. Photosynth Res 25: $147-150$

Vogelmann TC (1993) Plant tissue optics. Ann Rev Plant Physiol Plant Mol Biol 44:231-251 